\title{
$\omega$-3PUFAs Improve Cognitive Impairments Through Ser133 Phosphorylation of CREB Upregulating BDNF/TrkB Signal in Schizophrenia
}

\author{
Cuiping Guo ${ }^{1} \cdot \mathrm{Yi} \mathrm{Liu}^{1,2} \cdot$ Mao-sheng Fang ${ }^{3} \cdot$ Yuanyuan $\mathrm{Li}^{4} \cdot$ Wensheng $\mathrm{Li}^{1} \cdot$ Yacoubou Abdoul Razak Mahaman ${ }^{1,5}$. \\ Kuan Zeng ${ }^{1,3} \cdot$ Yiyuan $\mathrm{Xia}^{1} \cdot$ Dan Ke ${ }^{1} \cdot$ Rong Liu ${ }^{1} \cdot$ Jian-Zhi Wang ${ }^{1,6} \cdot$ Hui Shen ${ }^{4} \cdot$ Xiji Shu $^{7} \cdot$ Xiaochuan Wang ${ }^{1,6}$
}

Published online: 4 May 2020

(C) The American Society for Experimental NeuroTherapeutics, Inc. 2020

\begin{abstract}
Schizophrenia (SZ) is a serious mental condition and is associated with cognitive impairments. Brain-derived neurotrophic factor (BDNF) is one of the learning- and memory-related molecules found in the CNS and its level was reported to be reduced in SZ brain, while $\omega-3$ polyunsaturated fatty acids ( $\omega$-3PUFAs) could improve SZ symptoms, but its mechanism of action remains unknown. Using MK801 injection-induced SZ rat model, we here found that supplementation with $\omega$-3PUFAs improved the levels of p-CREB, BDNF, and p-TrkB in the brain of SZ rats, and restore hippocampal neuronal damage, thereby reducing cognitive impairments in SZ rats. However, overexpression of AAV9/CREB S133A (CREB inactivated mutation) downregulated BDNF/TrkB signaling pathway and remarkably abolished the preventive effect of $\omega$-3PUFAs in MK801-induced schizophrenia. Interestingly, AAV9/CREB S133D (CREB activated mutation) improved synaptic dysfunctions and cognitive defects in MK801 rats. In conclusion, these findings indicate that MK801-induced SZ lesions dephosphorylate CREB at Ser133 site, leading to neuron damage, and $\omega$-3PUFAs improve SZ cognitive impairments by upregulating the CREB/BDNF/TrkB pathway, which provides new clues for the mechanism of SZ cognitive impairments, and a basis for therapeutic intervention.
\end{abstract}

Key Words Schizophrenia $\cdot$ MK801 $\cdot \omega-3$ polyunsaturated fatty acids $\cdot$ cognitive impairments $\cdot$ CREB/BDNF/TrkB pathway

\section{Introduction}

Schizophrenia (SZ) is a common and serious mental health hazard and usually begins in adolescence or early adulthood [1-4], and it is highly recurrent, with high disabilities, and poor prognosis, and its etiopathogenesis is still unclear. SZ

Cuiping Guo and Yi Liu contributed equally to this work.

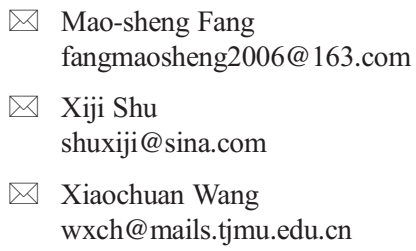

1 Department of Pathophysiology, School of Basic Medicine, Key Laboratory of Education Ministry of China for Neurological Disorders, Tongji Medical College, Huazhong University of Science and Technology, Wuhan 430030, China

2 Department of Pathophysiology, Weifang Medical University, Weifang 261053, China treatment and preventive measures are limited and impose a heavy burden on patients' families and society. A large number of studies have confirmed that SZ is associated with cognitive impairments, and this is more likely to affect the prognostic outcome than psychotic symptoms, which impairs the

3 Wuhan Mental Health Center, Wuhan 430022, China

4 Laboratory of Neurobiology, School of Biomedical Engineering, Tianjin Medical University, Tianjin, China

5 Cognitive Impairment Ward of Neurology Department, The Third Affiliated Hospital of Shenzhen University, 47 Youyi Rd., Shenzhen 518001, Guangdong Province, China

6 Co-innovation Center of Neuroregeneration, Nantong University, Nantong 226001, Jiangsu, China

Department of Pathology and Pathophysiology, School of Medicine, Jianghan University, Wuhan 430056, China 
professional social function of patients, with serious effects on the quality of life of patients $[5,6]$.

Brain-derived neurotrophic factor (BDNF) is a neurotrophic protein and together with its receptor is widely expressed in the nervous system [7, 8]. BDNF is transported by anterograde mean from the axoplasm to axon tip where it binds to its specific receptor, especially the most potent TrkB receptor, and causes TrkB autophosphorylation, which triggers signaling cascades that affect target tissues [9-11]. BDNF maintains neuronal survival, growth, differentiation, and migration of new neuronal precursor cells, and induces synaptic connections, to promote neuronal cell development. It was reported that during chronic stress damage in neurons during adulthood, BDNF helps to protect neurons, regulates calcium concentration and reduces the necrosis and apoptosis in hippocampal neurons, positively affects neuronal plasticity, and repairs. They also have been reported to be closely related to neuropsychiatric diseases, including SZ $[12,13]$.

In recent years, the role of nutritional factors in neuropsychiatric diseases has received increasing attention [14]. Among them, omega-3 polyunsaturated fatty acids $(\omega-$ 3PUFAs) are essential fatty acids for humans, including docosahexaenoic acid (DHA), eicosapentaenoic acid (EPA), and arachidonic acid (AA). These $\omega$-3PUFAs perform several important functions including cell membrane stabilization, gene expression regulation, cytokine and membrane protein balance maintenance, promoting growth and development, and many other properties like anti-cardiovascular disease, anti-inflammatory, and anticancer $[15,16]$. $\omega$-3PUFAs have become a hot research topic in the field of neurological disorders including neuropsychiatry because of its important influence on the development and function of the brain. $\omega$-3PUFAs can promote the expression of hippocampal neurogenesisrelated genes, regulate hippocampal neurogenesis, stimulate dendritic branches, activate related signaling pathways, and induce synaptic formation and long-term potentiation (LTP) $[17,18]$. In addition, $\omega$-3PUFAs can reduce neuronal oxidative stress and apoptosis, and play an important role in maintaining normal cognitive function [19]. Studies have found that $\omega$-3PUFAs can improve the symptoms of SZ [20,21], but the specific mechanism of the effect of $\omega$-3PUFAs on SZ cognition is not clear. Therefore, it is necessary to further explore the influence of $\omega$-3PUFAs on the cognitive function impairments related to SZ. Our previous study has shown that $\omega$ 3PUFAs enhances the CREB/BDNF/TrkB pathway [22]. Moreover, it has been reported that $\omega$-3PUFAs can upregulate CREB phosphorylation [23, 24], but the specific mechanism is unknown. To elucidate the mechanism of $\omega$-3PUFAs involvement in memory in SZ, we hypothesized that by promoting CREB phosphorylation, $\omega$ 3PUFAs may influence the CREB/BDNF/TrkB pathway.
We therefore here reported that $\omega$-3PUFAs improve BDNF/TrkB pathway alterations through Ser 133 phosphorylation of CREB in rat hippocampus and prevent MK801-induced cognitive impairments in rats. These provide new clues to the mechanism of SZ cognitive impairments, thereby providing an insight for therapeutic intervention.

\section{Material and Methods}

\section{Animals}

Male Sprague-Dawley rats ( 2 months old, $250 \pm 20$ g) were supplied by the Experimental Animal Central of Tongji Medical College, Huazhong University of Science and Technology. Rats were kept under standard laboratory conditions: 12-h light and 12-h dark with water and food ad libitum. Rats were randomly divided into groups and treated as stated in different parts of the study. All animal experiments were approved by the Institutional Animal Care and Use Committee at Tongji Medical College, Huazhong University of Science and Technology (IACUC Number: 2316).

\section{Stereotactic Surgery}

The rats were anesthetized with isoflurane and placed in a stereotaxic apparatus. After being sterilized with iodophors and $75 \%$ (vol/ $/ \mathrm{vol}$ ) alcohol, the scalp was incised along the midline between the ears. Holes were drilled in the hibateral skull stereotaxically at posterior $3.96 \mathrm{~mm}$, lateral $3.0 \mathrm{~mm}$, and ventral $3.0 \mathrm{~mm}$ relative to bregma. Using a microinjection system (World Precision Instruments), AAV-CMV-eGFPCREB S133D, AAV-CMV-eGFP-CREB S133A, or vector $\left(2 \mu \mathrm{L}, 2.0 \times 10^{12}\right.$ viral genomes per milliliter) was injected in the hippocampus CA1 region at a rate of $0.125 \mu \mathrm{L} / \mathrm{min}$; the needle was kept in place for $10 \mathrm{~min}$ before withdrawal, and the skin was sutured.

\section{Reagents}

$\omega$-3PUFAs are a deep sea fish oil capsule, the main ingredients of which are DHA $(\geq 110 \mathrm{mg})$ and EPA ( $\geq 170 \mathrm{mg})$. MK801 was purchased from Sigma Company (USA), MAP2 (1:250 millipore). For Western blotting, the primary antibodies used were CREB (1:1000; Cell Signaling Technology, USA), p-CREB (1:1000; Cell Signaling Technology, USA), BDNF (1:1000; Abcam, USA), TrkB (1:1000; Cell Signaling Technology, USA), p-TrkB (1:1000; Cell Signaling Technology, USA), and actin (1:1000; Abcam). 


\section{Behavior Tests}

\section{Open Field}

The open field is used to assess tension, anxiety, and exploration activities of experimental animals. The test equipment is a typical open field $\left(100 \times 100 \mathrm{~cm}^{2}\right.$ PVC square arena with 70 $\mathrm{cm}$-high walls). Rats were individually trained for a single 5min period. Anxiety is studied by analyzing the percentage of time spent in the middle of the arena. The total distance covered and central zone crossing were tracked and measured.

\section{Three Boxes Social Behavioral Experiments}

The experimental device consists of three boxes, each box with a size of $19 \times 45 \mathrm{~cm}^{2}$. The partition between each box is a transparent plexiglass with a channel in the middle to make three boxes open; in the left and right boxes, we placed a uniform metal cage in the center, enough to accommodate a rat. Before the experiment, the rats were taken in the behavior test room for half an hour; we put a stranger rat into the metal cage in one side of the box, while the metal cage on the other side of the box is empty. The three boxes are separated by a transparent glass resin plate, and the test rat is placed in the box on the other side for $5 \mathrm{~min}$. The glass resin board separating the boxes is then removed so that the test rat can move freely in three boxes for $5 \mathrm{~min}$. The number of exchanges and residence time between the rats and the stranger rat was recorded.

\section{High Plus Maze Test}

High plus maze consists of two opposite open arms, two opposite closed arms, and a central zone. The maze size is arm length: $425 \times$ arm width $120 \times$ arm height $225 \mathrm{~mm}$. At the beginning of the experiment, the rats were placed into the maze from the central grid facing the closed arm, and the activity within $5 \mathrm{~min}$ was recorded. Observation indicators include open arm entry times, open arm dwell time, closed arm entry times, and closed arm dwell time.

\section{Novel Objective Recognition Test}

The rats were taken to the new object recognition room $24 \mathrm{~h}$ before the test, then we put the rat into a $100 \mathrm{~cm} \times 100 \mathrm{~cm} \times$ $100 \mathrm{~cm}$ plastic container for $5 \mathrm{~min}$, then took it back to the arena from the same starting point; they were allowed for 5 min to be familiar with A and B objects. After each familiar period, the arena and objects were cleaned with $75 \%$ ethanol. Two hours after the familiarization period, the B object was replaced by the $\mathrm{C}$ object, and the rats were given $5 \mathrm{~min}$ to explore the two objects. After $24 \mathrm{~h}$, the $\mathrm{C}$ object was replaced with a D object, and the rat was given 5 min to explore two objects.

\section{Morris Water Maze Test}

Spatial learning and memory were tested by MWM as described in a previous study. For spatial learning, the rats were trained in the water maze to find a hidden platform for 5 days, four trials per day from 8:00 am to $14: 00 \mathrm{pm}$. In each trial, the rats started from one of four quadrants facing the wall of the pool and ended when the animal climbed on the platform. If the rats failed to find the platform within $60 \mathrm{~s}$, they were guided to the platform and allowed to stay on the platform for $20 \mathrm{~s}$ uniformly. The swimming path and the time used to find the platform (latency) were recorded by a tracking video camera. Spatial memory was tested the next day after training. The platform was removed and the latency of first crossing of the platform, the number of crossing, and the time in target quadrant were recorded for $60 \mathrm{~s}$.

\section{Western Blotting}

The hippocampus was rapidly removed and homogenized at $4{ }^{\circ} \mathrm{C}$, using a buffer containing $50 \mathrm{mmol} / \mathrm{L}$ Tris- $\mathrm{HCl}, \mathrm{pH} 7.4$, $150 \mathrm{mmol} / \mathrm{L} \mathrm{NaCl}, 10 \mathrm{mmol} / \mathrm{L} \mathrm{NaF}, 1 \mathrm{mmol} / \mathrm{L} \mathrm{Na}_{3} \mathrm{VO}_{4}$, $5 \mathrm{mmol} / \mathrm{L}$ EDTA, $2 \mathrm{mM}$ benzamidine, and $1 \mathrm{mM}$ PMSF, while the primary hippocampal neurons were extracted on ice in radio immunoprecipitation assay (RIPA) buffer. After centrifugation of the tissue homogenates or cell lysate at $12000 \mathrm{rpm} / \mathrm{min}$, the supernatants were collected. Protein concentrations were quantified by bicinchoninic acid (BCA) protein kit (Pierce, Rockford, IL, USA). Proteins were separated by SDS-polyacrylamide gel electrophoresis $(10 \%$ gel $)$ and transferred to nitrocellulose membranes. After immersion in $5 \%$ skim milk at $25^{\circ} \mathrm{C}$ for $1 \mathrm{~h}$, the membranes were incubated overnight with the primary antibody at $4{ }^{\circ} \mathrm{C}$. Then, the imprinting was conjugated to IRDye TM $(800 \mathrm{CW})$ anti-mouse or anti-rabbit $\operatorname{IgG}$ for $1 \mathrm{~h}$ at $25^{\circ} \mathrm{C}$, and the image was viewed with an Odyssey infrared imaging system (LI-COR Biosciences, USA).

\section{Primary Hippocampal Neuron Culture}

Primary hippocampal neurons were prepared from 17 to 18 days old Sprague-Dawley rat embryos. The hippocampus was dissected and gently chopped in Hank's buffered saline solution and then suspended in a $0.25 \%(v / v)$ trypsin solution for 15 min at $37{ }^{\circ} \mathrm{C}$. Neurons were plated in 6-well and 12well plates coated with $100 \mu \mathrm{g} / \mathrm{mL}$ poly-D-lysine and supplemented with $2 \%(v / v)$ B-27 and $1 \times$ GlutaMAX. The neurons were cultured for 9 days and then treated with MK801, AAV9/CTR, AAV9/CREB-S133D, and AAV9/CREBS133A. At the end of the treatments, cells were collected 
and lysed in RIPA buffer for further biological detections or fixed with $4 \%$ paraformaldehyde for immunofluorescence imaging. All cell culture reagents were purchased from Thermo Fisher Scientific. Sholl analysis was used to measure dendritic complexity as reported previously [25]. Dendritic length was analyzed and measured using semi-automatized protocol via Imaris software (Bitplane, Inc.).

\section{BDNF ELISA Assay}

The brain and hippocampus primary neurons were lysed in RIPA buffer and centrifuged at $3000 \times g$ for $10 \mathrm{~min}$ at $4{ }^{\circ} \mathrm{C}$, and the supernatant containing BDNF was collected. The RIPAavailable BDNF levels were determined by ELISA according to the procedure provided by the assay kit manufacturer.

\section{Electrophysiology}

Slice Preparation The rats were perfused with $20 \mathrm{ml}$ ice cold solution of aCSF containing (in $\mathrm{mM}$ ) $120 \mathrm{NaCl}, 2.5 \mathrm{KCl}, 2$ $\mathrm{CaCl}_{2} \cdot 2 \mathrm{H}_{2} \mathrm{O}, 1.25 \mathrm{KH}_{2} \mathrm{PO}_{4}, 2 \mathrm{MgSO}_{4} \cdot 7 \mathrm{H}_{2} \mathrm{O}, 26 \mathrm{NaHCO}_{3}$, and 10 glucose, saturated with $95 \% \mathrm{O}_{2}$ and $5 \% \mathrm{CO}_{2}$ and buffered to a $\mathrm{pH}$ of 7.4. Following sectioning at $360-\mu \mathrm{m}$ thickness, the slices were incubated in oxygenated aCSF at $32{ }^{\circ} \mathrm{C}$ to recover for $40 \mathrm{~min}$ and at $20-25{ }^{\circ} \mathrm{C}$ to recover for $1 \mathrm{~h}$. Then slices were transferred to the recording chamber with continuous oxygenated aCSF perfusion.

Recording of LTP For LTP, acute brain sections were transferred to a recording chamber and submerged in aCSF. The sections were placed in a chamber with an $8 \times 8$ microelectrode array (Parker Technology, Beijing, China) in the bottom plane (each $50 \times 50 \mathrm{~mm}$ size with an interelectrode distance of $150 \mu \mathrm{m})$ and kept submerged in the aCSF. Stimulation signals were provided by the MED64 system (Alpha MED Sciences, Panasonic). Field excitatory postsynaptic potential (fEPSP) in CA1 neurons was recorded by stimulating CA3 neurons. LTP was induced by applying three columns of high-frequency stimulation (HFS; $100 \mathrm{~Hz}, 1$-s duration). The LTP magnitude was quantified as a percentage change in the fEPSP slope taken during the $60 \mathrm{~min}$ interval following LTP induction.

Recording of AMPAR sEPSCs Hippocampal slices were held between nylon net in an interface chamber and then fully submerged in the aCSF $(3 \mathrm{ml} / \mathrm{min})$ at room temperature $(20$ $25^{\circ} \mathrm{C}$ ). Pyramidal cells in the CA1 hippocampal slice were identified by a DIC-infrared upright microscope. Glass microelectrodes (4-6 M $\Omega$ ) filled with an internal solution contained (in mM) $140 \mathrm{~K}$-gluconate, $2 \mathrm{MgCl}_{2}, 10 \mathrm{HEPES}, 10$ BAPTA, 2 $\mathrm{Mg}_{2} \mathrm{ATP}, 0.5 \mathrm{CaCl}_{2}-\mathrm{H}_{2} \mathrm{O}, 0.5 \mathrm{Li}-\mathrm{GTP}, \mathrm{pH} 7.2$, and $290 \mathrm{mOsm}$. Recordings were carried out at $-60 \mathrm{mV}$. Five millimolar QX314 was added to block voltage-gated $\mathrm{Na}^{+}$channels and GABAB receptors. The sEPSCs of AMPAR were recorded in the presence of $100 \mu \mathrm{M}$ picrotoxin (PTX) and $50 \mu \mathrm{M} \mathrm{dL}-2$ amino-5-phosphonovaleric acid (D-APV).

Paired Pulse Ratio Paired pulse ratios were calculated as a ratio of EPSC2 to EPSC1 separated by inter-stimulus intervals of 20, 50, 100, 150, and $200 \mathrm{~ms}$ for EPSCs. To measure the input-output curves for pyramidal neurons, a bipolar tungsten electrode was place to the stratum radiatum with a bipolar tungsten electrode $\sim 50 \mu \mathrm{m}$ from the recording electrode in order to test potential presynaptic effects.

Recording of NMDAR/AMPAR Ratio To record evoked EPSCs, pipette electrodes with a resistance of 3-5 M $\Omega$ were filled with the internal solution containing (in $\mathrm{mM}$ ) $135 \mathrm{CsMeSO}_{4}, 8 \mathrm{NaCl}, 10 \mathrm{HEPES}, 0.25$ EGTA, 2 $\mathrm{Mg}_{2} \mathrm{ATP}, 0.3 \mathrm{Na}_{3} \mathrm{GTP}, 0.1$ spermine, and 7 phosphocreatine $(\mathrm{pH}$ 7.25-7.3). The AMPAR- and NMDARdependent synaptic responses were evoked by a bipolar stimulation electrode (FHC) that was located $\sim 50 \mu \mathrm{m}$ rostral to the recording electrode in the same layer. In the presence of $100 \mu \mathrm{M}$ PTX, we obtained stable synaptic responses at a holding potential $-60 \mathrm{mV}$; the amplitude of these responses represents the AMPAR-specific component. At $+40 \mathrm{mV}$, an amplitude of $100-\mathrm{ms}$ post-stimulus was identified as the NMDA-specific response.

\section{Golgi Staining}

The rats were anesthetized by isoflurane and transcardially perfused with proximately $300 \mathrm{ml}$ of normal saline containing $0.5 \%$ sodium nitrite, followed with $300 \mathrm{ml}$ of $4 \%$ formaldehyde solution and then $500 \mathrm{ml}$ staining solution (5\% chloral hydrate, $5 \%$ potassium dichromate, and $4 \%$ formaldehyde) over $2 \mathrm{~h}$. Brains were removed from the skull and incubated in the staining solution for 3 days and in $1 \%$ silver nitrate solution for another 3 days in the dark. The silver nitrate solution was changed every day. Finally, the brains were sliced using a vibrate microtome (Leica, Wetzlar, Germany) at a thickness of $100 \mu \mathrm{m}$.

\section{TUNEL Assay}

Cellular DNA fragmentation in either DMSO vehicle control or MK801-treated primary hippocampal neurons was determined using TUNEL assay. Primary hippocampal neurons were fixed in $4 \%$ paraformaldehyde for $30 \mathrm{~min}$, then permeabilized in $0.5 \%$ Triton X-100 in phosphate-buffered saline (PBS) for $15 \mathrm{~min}$. TUNEL staining was performed using a fluorescein in situ cell death detection kit (Dalian Meilun Biotechnology Co., Ltd., Product ID: MA0224) according to the manufacturer's instructions. 


\section{Statistical Analysis}

All experiments were repeated at least three times. Data were expressed as mean \pm SEM and analyzed using graphPad prism statistical software. The one-way ANOVA was used to determine the differences among groups. For the comparison between two groups, the Student's $t$ test was used. The significance was assessed at $p<0.05$.

\section{Results}

\section{MK801-Induced Schizophrenia-Like Cognitive Impairments and Synaptic Dysfunction}

Thirty healthy SD rats weighing $250 \pm 20$ g were adaptively domesticated for 7 days in the animal laboratory under the following conditions: $22-24{ }^{\circ} \mathrm{C}$; light/dark cycle of $12 \mathrm{~h}$. Then the animals were randomly divided into 2 groups (control and model MK801, regarded as a surrogate for the positive symptoms of schizophrenia [26-28]) of 15 rats each. The rats in the model group were daily injected intraperitoneally with MK801for 1 week $(0.3 \mathrm{mg} / \mathrm{kg})$, when saline served as control for 1 week (Fig. 1A). We first carried open-field test after injection and the result showed that there was no significant difference in the total distance covered (Fig. 1B) between the two groups, but the number of times in the zone crossing is significantly reduced in the MK801 rats compared with the control (Fig. 1C), implicating that MK801 might induce anxiety. To further explore anxiety in the rats, we employed high plus maze test and found that the MK801 rats have shortened entries to both open and closed arm (Fig. 1D, E) when compared with the control ones, strongly supporting SZ-like anxiety in MK801 rats. To examine the social interaction of the rats, we carry out the three boxes behavioral test and found out that the social ability was significantly impaired in the model group (Fig. 1F). The novelty recognition experiment allows to explore short-term memory capacity; the results from this experiment showed that in the MK801 group the curiosity toward exploring new things were significantly reduced, as the time spent for exploration of new object in $2 \mathrm{~h}$ and $24 \mathrm{~h}$ were significantly decreased
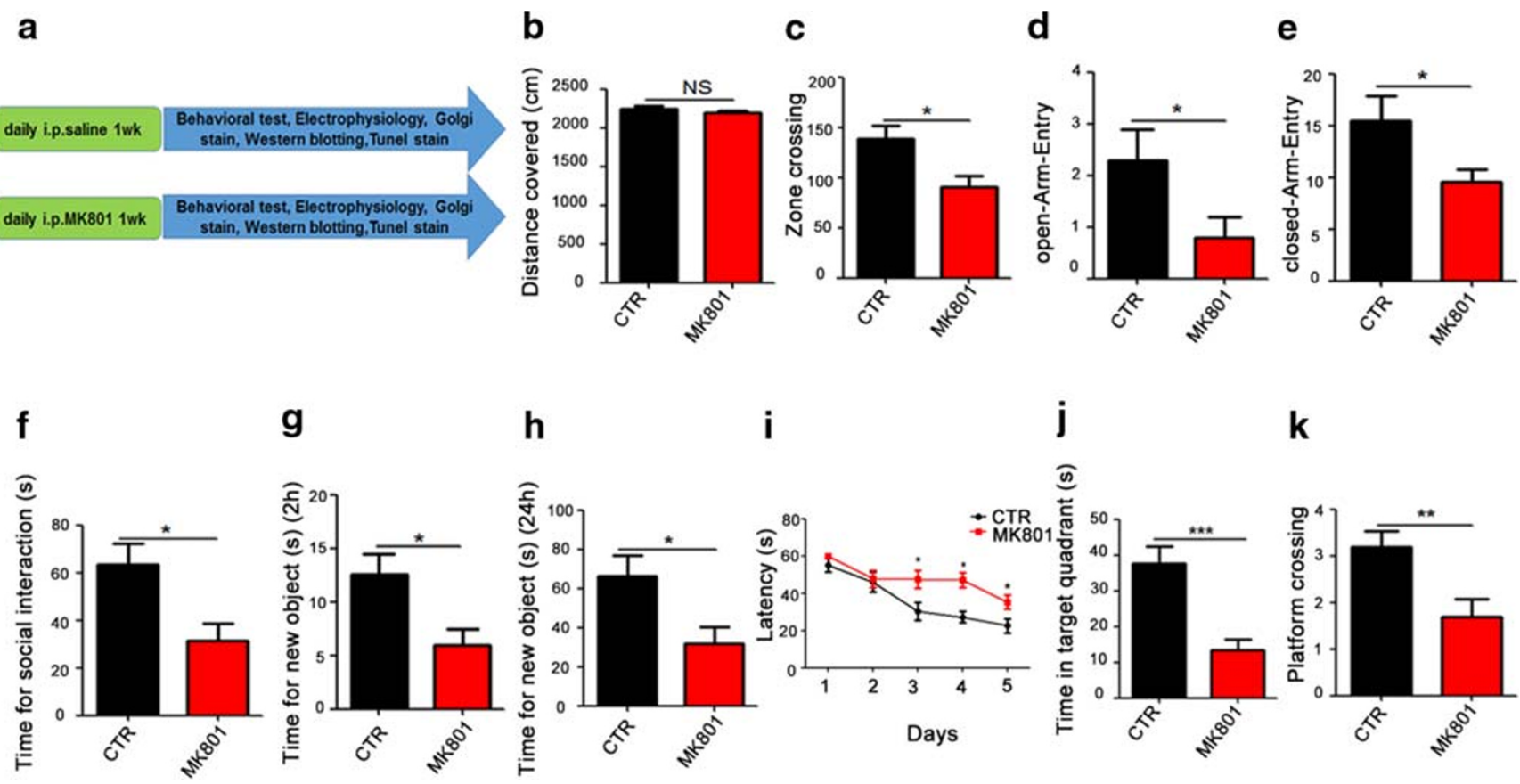

k

Fig. 1 MK801-induced schizophrenia damaged cognitive impairments. (A) Experimental design sketch. Thirty healthy SD rats were randomly divided into 2 groups (control and model MK801). The rats in the model group were daily injected intraperitoneally with MK801for 1 week $(0.3 \mathrm{mg} / \mathrm{kg})$, when saline served as control for 1 week. Following the treatment, behavioral, electrophysiological, and biochemical tests were performed. (B, C) The open field showed no difference in the total distance covered (B), but a significant decrease in the zone crossing (C) in the MK801 treated group. (D, E) High plus maze test showed the number of the open-arm-entry (D) and the closed-arm-entry (E). (F) In the three

boxes social behavior experiment, the time spent exploring the new or stranger rat was recorded. $(\mathrm{G}, \mathrm{H})$ Novel object recognition test (NOR) showed the measured recognition time of the new object in $2 \mathrm{~h}(\mathrm{G})$ and $24 \mathrm{~h}(\mathrm{H})$. (I-K) The Morris water maze test: the latency to find the hidden platform from day 1 to day 5 (I), the spatial memory was tested by removing the platform, and the time spent in the target quadrant $(\mathrm{J})$ and number of the position of target platform crossing (K) were measured. Data are presented as mean \pm SEM. $* p<0.05, * * p<0.01, * * * p<0.001$, versus control group 
a

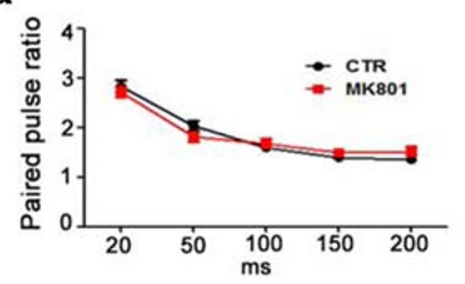

e

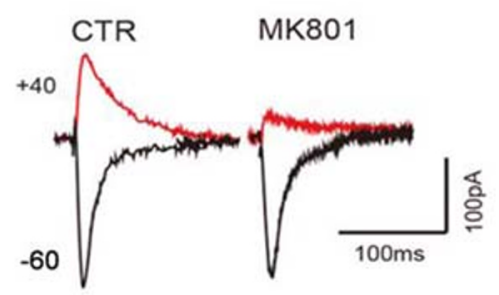

i
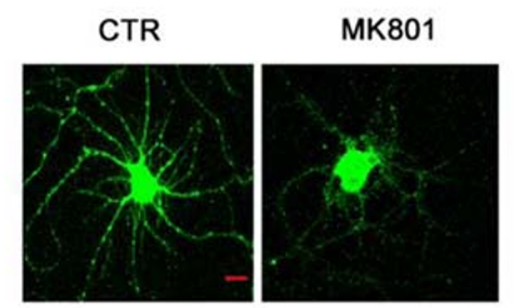

Fig. 2 MK801-induced schizophrenia damaged synaptic plasticity. (A) The graph of representative records of paired pulsed ratio (PPR) test in hippocampal slices. For pairwise pulse ratio experiments, paired stimuli ( $25 \mathrm{~ms}, 50 \mathrm{~ms}, 100 \mathrm{~ms}, 150 \mathrm{~ms}, 200 \mathrm{~ms}$ ) were given and the response were recorded; $n=9$ cells from 5 rats for each of the MK801 and control groups. (B-D) The representative images of short trace recordings from representative cells of AMPA-sEPSCs (B). Frequency (C) and amplitude (D) of AMPA-sEPSCs are unchanged in neurons from both groups. (E, F) Ratio of NMDAR- to AMPAR-mediated EPSCs is significantly decreased by MK801. Representative EPSCs recorded at $-60 \mathrm{mV}$ and +

(Fig. 1G, H). Finally, we tested memory and learning abilities using Morris water maze (MWM), and observed that the MK801 rats showed significantly increased latency to find the hidden platform (Fig. 1I) compared with the control group. On day 6, the spatial memory was tested by removing the platform. A remarkable decrease in the time spent in the target quadrant (Fig. 1J), as well as a decrease in the number of target platform crossing (Fig. $1 \mathrm{~K})$, was observed in the SZ rats. Together, these data demonstrate that MK801-induced SZ could cause anxiety, social interaction alteration, and learning and memory impairments in rats.

It is clear that MK801-induced SZ leads to a series of behavioral dysfunctions in rats. Among these behavioral alterations, learning and memory impairments are commonly shared with other neurological disorders including
C

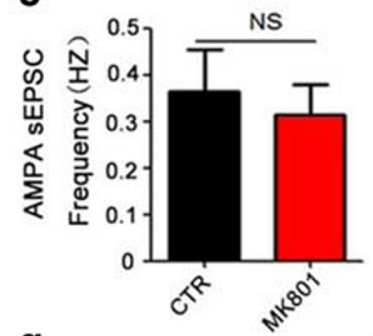

g

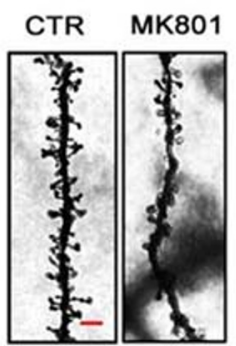

h
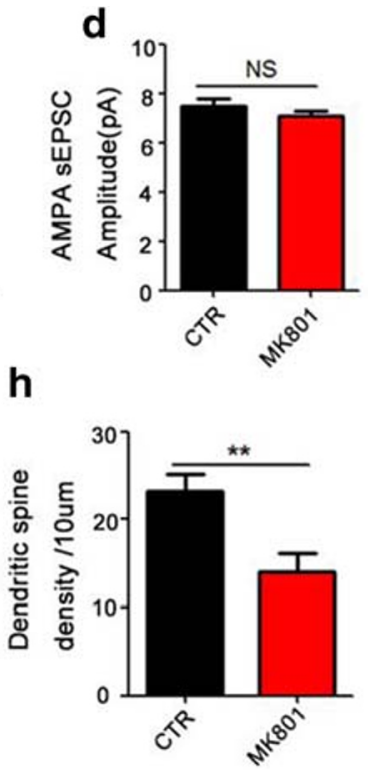

k

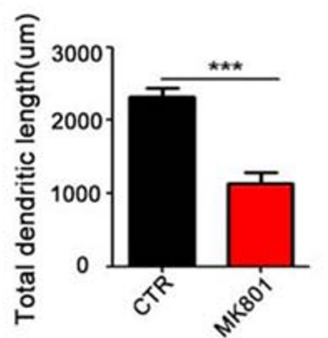

$40 \mathrm{mV}(\mathrm{E})$ and the quantification $(\mathrm{F})$ are shown; $n=11$ cells from 5 rats for each of the control and MK801 rat groups. $(\mathrm{G}, \mathrm{H})$ Representative images of dendritic spines of neurons from Golgi impregnated hippocampus (G); averaged spine density (mean spine number per $10-\mathrm{mm}$ dendrite segment) was measured in rats $(\mathrm{H})$ Scale bar $=2 \mu \mathrm{m}$. (I-K) Rats' primary hippocampal neurons at DIV 9 were treated with DMSO for control and MK801 for model for $48 \mathrm{~h}$. Representative images after treatment (I, Scale bar $=10 \mu \mathrm{m})$, Sholl analysis $(\mathrm{J})$, quantitative analyses of dendritic length $(\mathrm{K}), n=16$ hippocampal neurons. All data represent mean $\pm \mathrm{SEM}$. $* p<0.05, * * p<0.01$, and $* * * p<0.001$ versus control

neurodegenerative diseases like Alzheimer [29, 30]. The hippocampus is an integral part of the limbic system [31] and is known to play a crucial role in memory and spatial localization which is the reflection of synaptic plasticity [32]. As learning and memory were found to be impaired in this model, we wonder whether MK801-induced SZ might alter synaptic plasticity. To further explore how the synaptic transmission in the CA3-CA1 Schaffer collateral (SC) is impaired, paired pulsed ratio (PPR) which is a form of short-term synaptic plasticity was evaluated, and the results showed that the PPR of the two groups of rats were not obviously different, suggesting that presynaptic vesicle release was not affected (Fig. 2A). We then examined the effect of MK801-induced SZ on AMPAR-mediated spontaneous excitatory postsynaptic currents (AMPAR-sEPSCs) (Fig. 2B). Neither the frequency nor the amplitude of sEPSCs was affected by MK801 (Fig. 
a

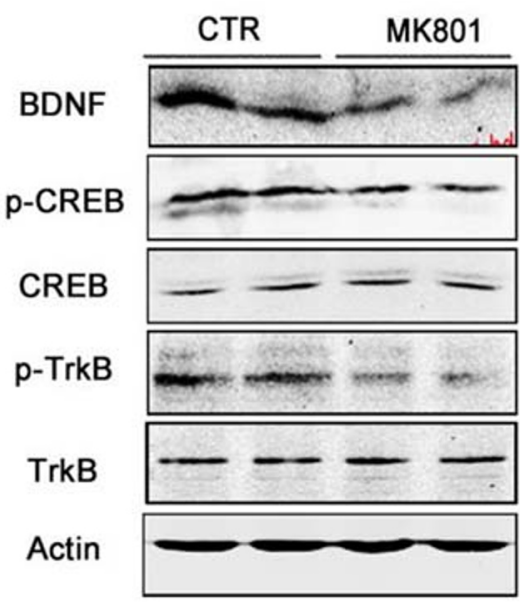

b

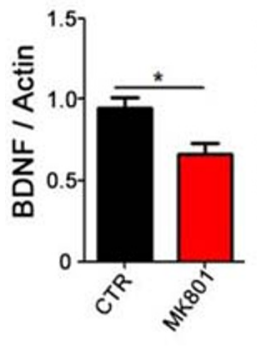

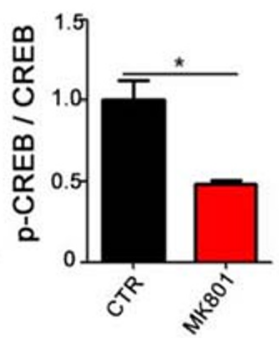

d

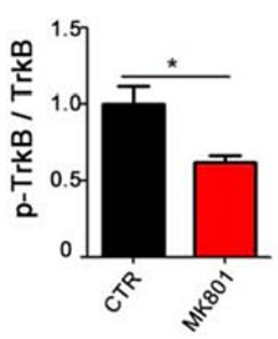

i

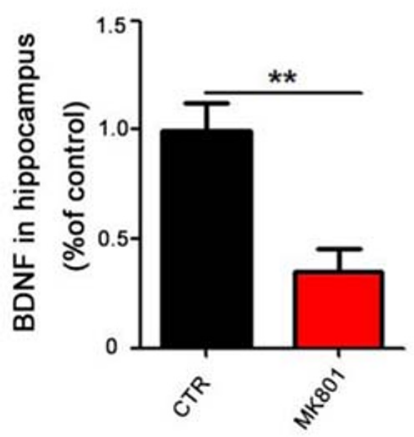

j

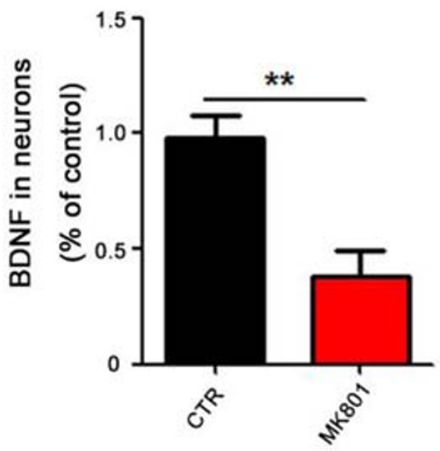

e

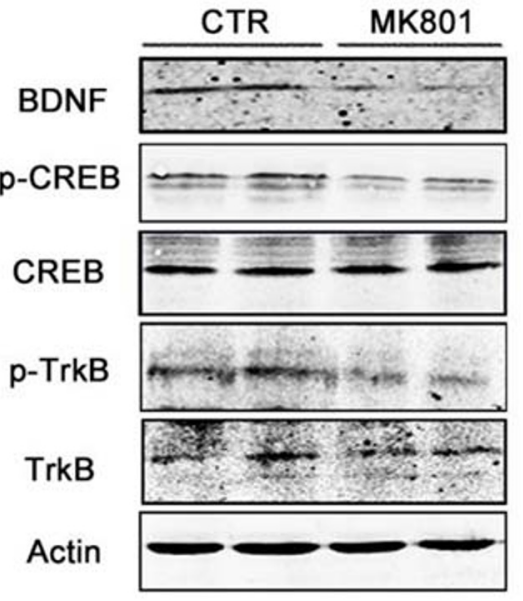

g
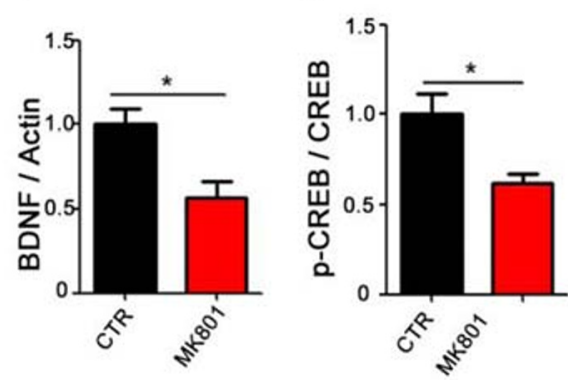

h

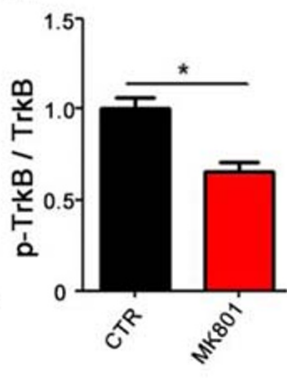

k

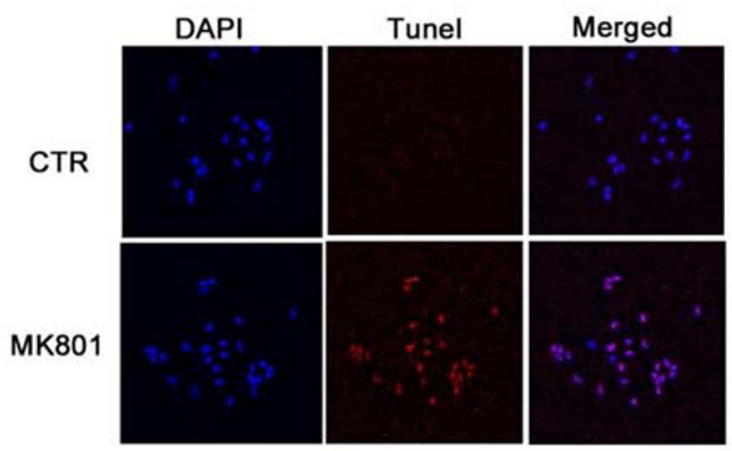

Fig. 3 MK801-induced hippocampal CREB/BDNF/TrkB pathway alteration in rat brains and in primary hippocampal neurons. (A-D) Brain tissues (hippocampus) from model group and control group were homogenized, and $\mathrm{p}-\mathrm{TrkB}$, TrkB, $\mathrm{p}-\mathrm{CREB}, \mathrm{CREB}$, and BDNF protein levels were detected by immunoblotting. Actin was used as a loading control (A). Quantitative analysis of the BDNF (B), p-CREB (C), and p-TrkB (D) levels. (E-H) Primary hippocampal neurons from MK801treated and control cells were homogenized, and p-TrkB, TrkB, p-CREB,

2C, D). To explore whether MK801-induced SZ influences basal NMDAR-mediated synaptic transmission, we calculated the NMDAR/AMPAR ratio (Fig. 2E). Interestingly, this ratio was significantly lower in the CA1 pyramidal cells from MK801-induced SZ rats than in the control ones (Fig. 2F), suggesting impaired synaptic function in SZ rats. To
CREB, and BDNF protein levels were detected by immunoblotting (E). Actin was used as a loading control. Quantitative analysis of the BDNF (F), p-CREB (G), and p-TrkB (H) levels. $n=4$ for both control model groups. (I, J) The ELISA to determine the level of BDNF in hippocampus tissue (I) and primary hippocampal neurons (J). (K) TUNEL staining to determine neuronal apoptosis by the high fluorescence of apoptotic bodies staining. Data are presented as mean $\pm \mathrm{SEM} ;{ }^{*} p<0.05$ and ${ }^{* *} p<0.01$ versus control group

investigate the underlying mechanism based on morphology, we further examined the spine density of hippocampal neurons (Fig. 2G). The result revealed a significant decrease in the dendritic spine density of the SZ rats (Fig. 2H) compared with the control ones. Furthermore, we examined the dendritic morphology of hippocampal primary neurons following 

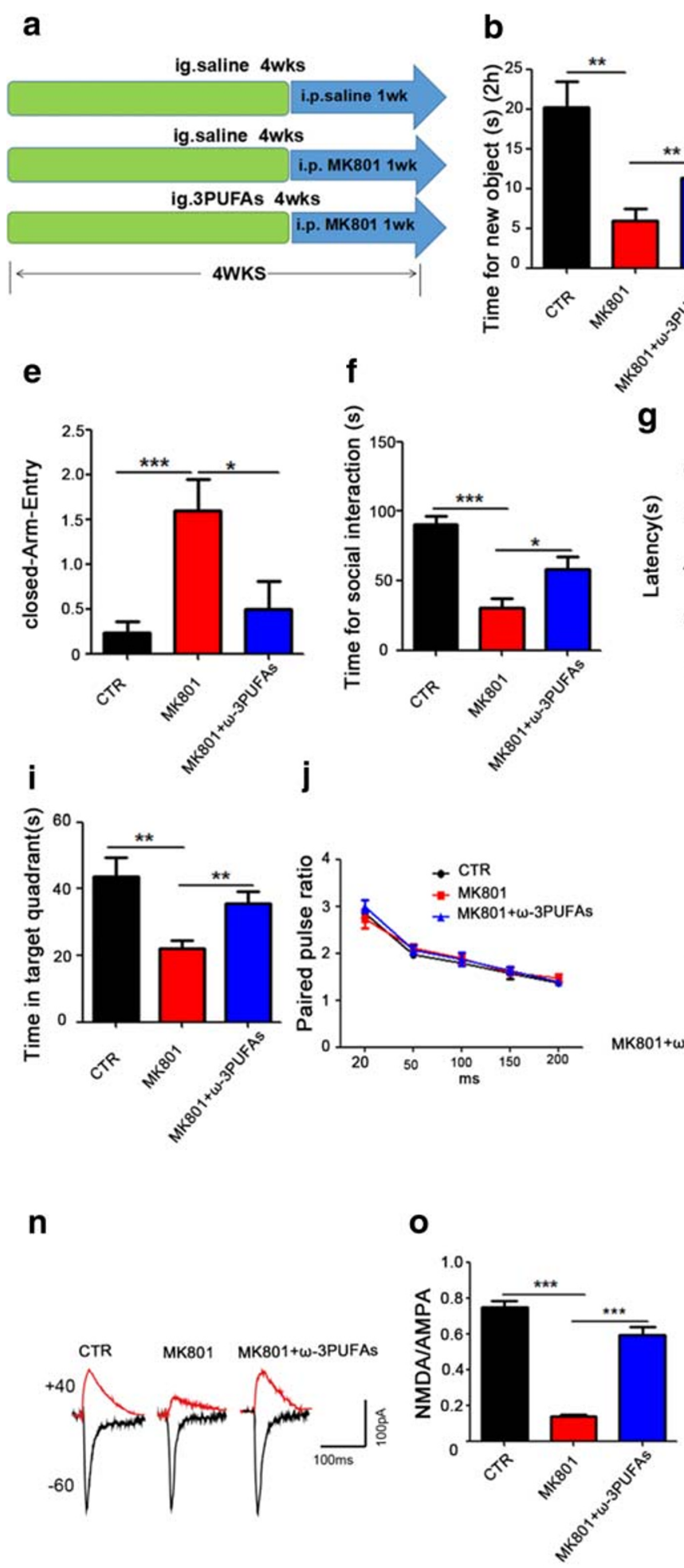

treatment with MK801 by using anti-MAP2 antibody (Fig. 2I). When compared with the control, $1 \mu \mathrm{M}$ MK801 resulted in an obvious decreased dendritic arborization complexity at all points farther than $40 \mu \mathrm{m}$ from the cell body (Fig. 2J, K). These findings suggest that MK801/SZ-induced cognitive impairments are associated with synaptic dysfunction.

\section{C}

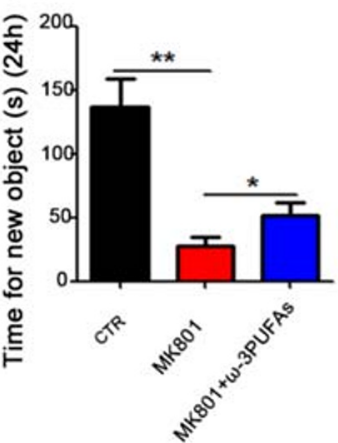

g

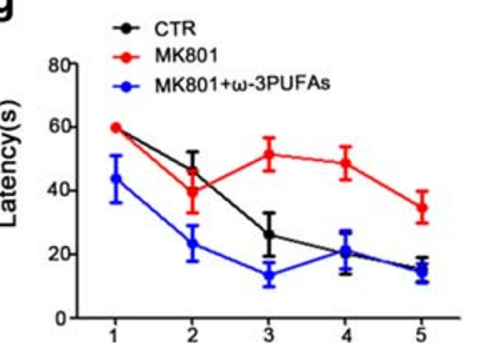

k

CTR

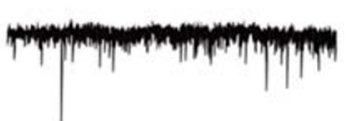

мК801

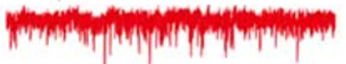

h

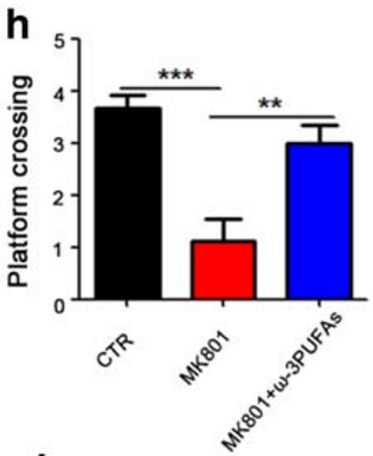

I

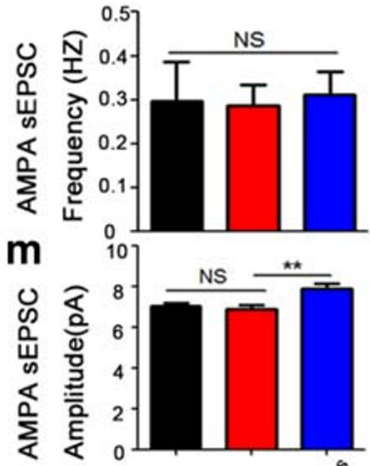

q

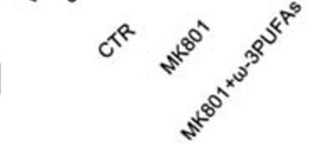

p
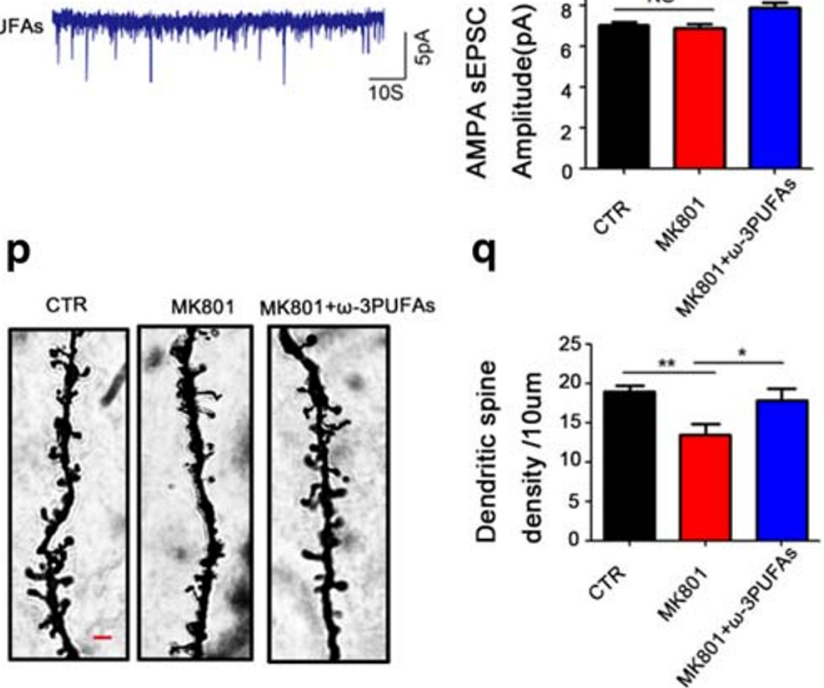

d

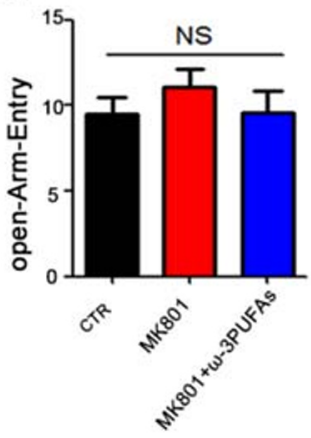

MK801-Induced Hippocampal CREB/BDNF/TrkB Pathway Alteration in Rat Brains and in Primary Hippocampal Neurons

Davide Carlino et al. [13] have reported that BDNF is associated with SZ cognitive dysfunction. When BDNF binds to 
4 Fig. $4 \omega$-3PUFAs prevented MK801-induced SZ cognitive deficits and hippocampal neuronal plasticity damage. (A) Experimental design sketch. Forty-five healthy SD rats were randomly divided into 3 groups as control (Ctr), model (Mod), and preventive (Pre). In the control group, 15 rats were orally administered with normal saline for 4 weeks, while in the model group another 15 rats were intraperitoneally daily injected with MK801 $(0.3 \mathrm{mg} / \mathrm{kg})$ for 1 week. In the preventive group, 15 rats were first intragastrically administered with deep sea fish oil $(400 \mathrm{mg} / \mathrm{kg}$ of $\omega$ 3PUFAs) for 4 weeks and then intraperitoneally daily injected with MK801 $(0.3 \mathrm{mg} / \mathrm{kg})$ for last 1 week. Following the treatment, behavioral, electrophysiological, and biochemical tests were performed. (B, C) Novel object recognition test (NOR) showed the measured time spent with the new object in $2 \mathrm{~h}(\mathrm{~B})$ and $24 \mathrm{~h}(\mathrm{C})$. (D, E) High plus maze test showed the number of open-arm-entry (D) and the closed-arm-entry (E). (F) Three boxes of social behavioral experiment showed the time spent in the box of stranger rat. (G-I) Morris water maze test: the latency to find the hidden platform from day 1 to day $5(\mathrm{G})$, the spatial memory was tested by removing the platform, and the number of the position of the target platform crossings $(\mathrm{H})$ and time spent in the target quadrant (I) were recorded. (J) The graph of representative records of paired pulsed ratio (PPR) test in hippocampal slices. For pairwise pulse ratio experiments, paired stimuli ( $25 \mathrm{~ms}, 50 \mathrm{~ms}, 100 \mathrm{~ms}, 150 \mathrm{~ms}, 200 \mathrm{~ms}$ ); $n=10$ cells from 4 rats for control group, $n=11$ cell from 4 rats for preventive group, and $n=11$ cells from 4 rats for the model group. (K-M) The representative images of short traces recordings from representative cells of AMPA-sEPSCs (K). Frequency (L) and amplitude (M) of AMPA-sEPSCs were recorded in neurons from both the control and mod groups. Ratio of NMDAR- to AMPAR-mediated EPSCs was significantly increased by $\omega$-3PUFAs. Representative EPSCs recorded at $-60 \mathrm{mV}$ and $+40 \mathrm{mV}(\mathrm{N})$ and the quantification $(\mathrm{O})$ are shown; $n=12$ cells from 4 rats for preventive group, $n=12$ cells from 4 rats for model group and 12 cells from 3 rats for control group. (P, Q) Representative dendritic spines of neurons from Golgi-impregnated hippocampus (P), averaged spine density (mean spine number per $10-\mathrm{mm}$ dendrite segment) was measured in rat $(\mathrm{Q})$. Scale bar $=2 \mu \mathrm{m}$. Data are presented as mean \pm SEM; $* p<0.05, * * p<0.01$, and $* * * p<0.001$ versus model

TrkB, it activates the intracellular region of this receptor and leads to enhanced TrkB autophosphorylation [33, 34], which in turn activates the Ras-MAPK pathway, and finally activates CREB by inducing its phosphorylation at the Ser133 site. Upon activation, CREB promotes neuronal survival and increases synaptic plasticity and neurogenesis by increasing the expression of the BDNF gene and the anti-apoptotic protein gene BCL-2 [35]. In view of this, we speculated that the CREB/BDNF/TrkB pathway may be involved in the cognitive alterations seen in SZ. To elucidate this hypothesis, we performed Western blotting and the result from the MK801induced SZ (Fig. 3A) showed a significant reduction in the protein levels of BDNF, p-CREB, and p-TrkB (Fig. 3B-D). Similarly, Western blotting from hippocampal primary neurons treated with MK801 showed similar results (Fig. 3E$\mathrm{H})$. We also used ELISA to determine the level of BDNF and found out that the BDNF level was decreased in both hippocampal (Fig. 3I) and primary neuron (Fig. 3J) lysates from MK801-treated group. This result was supported by TUNEL staining where MK801 triggers neuronal apoptosis, as suggested by the high fluorescence of apoptotic body staining in the MK801 treatment group when compared with the control (Fig. 3K), implying that this effect might be associated with the decrease in the BDNF level in the brain and supporting that the lack of BDNF in the brain may stimulate signaling cascades that lead to neuronal apoptosis. Together, these data from rat and primary neurons imply that MK801induced $\mathrm{SZ}$ causes CREB/BDNF/TrkB pathway dysfunction and BDNF deficiency.

\section{w-3PUFAs Restored MK801-Induced SZ-Like Behavioral Alterations and Prevented Hippocampal Neuronal Damage}

The lack of $\omega$-3PUFAs has been identified as an environmental risk factor for SZ. Studies have found that $\omega$-3PUFAs can improve the symptoms of SZ, but the molecular mechanisms underlying the effects of $\omega$-3PUFAs on SZ cognition deficits remain unknown. To explore the mechanism of $\omega$-3PUFA effects in SZ, 45 healthy SD rats were randomly divided into 3 groups as control (Ctr), model (Mod), and preventive (Pre). In the control group, 15 rats were orally administered with normal saline for 4 weeks, while in the model group another 15 rats were intraperitoneally daily injected with MK801 $(0.3 \mathrm{mg} / \mathrm{kg})$ for 1 week. In the preventive group, 15 rats were first intragastrically administered with deep sea fish oil ( $400 \mathrm{mg} / \mathrm{kg}$ of $\omega$-3PUFAs) for 4 weeks and then intraperitoneally daily injected with MK801 $(0.3 \mathrm{mg} / \mathrm{kg})$ for last 1 week (Fig. 4A). Following the treatment, we performed several behavioral tests. The results from the novel object recognition (NOR) test showed that in the Pre group, the curiosity of exploring new things was significantly higher when compared with the Mod group, as the time spent exploring new object during a $2 \mathrm{~h}$ and $24 \mathrm{~h}$ tests were significantly increased (Fig. 4B, C). High plus maze test showed that there is no difference in open arm time among the groups (Fig. 4D), while the time spent in the closed arm in the Pre group was markedly decreased as compared with the Mod group (Fig. 4E), implicating that $\omega$-3PUFAs reduces MK801-induced anxiety. The three boxes social experiments showed that the Pre group's social ability was significantly restored compared with that of MK801 rats (Fig. 4F). In the Morris water maze, the Pre group showed a significantly decreased latency to find the hidden platform compared with the Mod group (Fig. 4G), while the target platform crossing (Fig. 4H) and time spent in the target quadrant (Fig. 4I) were remarkably increased, suggesting the restoration of spatial memory. Taken together, these data demonstrate that $\omega$-3PUFAs attenuate MK801-induced SZ rat anxiety, social ability impairments, and learning and memory deficits.

To investigate the mechanism underlying the role of $\omega$ 3PUFAs in preventing behavioral alterations, we wonder whether $\omega$-3PUFAs play a role in synaptic plasticity, and carried out some electrophysiology and neuronal integrity tests 
from hippocampal slices after behavioral tests. The PPR results showed no obvious difference between the groups (Fig. $4 \mathrm{~J})$, suggesting that it does not affect presynaptic vesicle release. Interestingly, when we examined the AMPAR-sEPSCs (Fig. 4K-M), no difference was observed in its frequency among all groups (Fig. 4L); however, the amplitude of sEPSCs, even though not different comparing with the control, was significantly enhanced in the Pre group when compared with the Mod (Fig. 4M). To explore whether $\omega$-3PUFA has an effect on the basal NMDAR-mediated synaptic transmission, we calculated the NMDAR/AMPAR ratio (Fig. 4N), and found out that this ratio was significantly increased in the Pre group than in the model SZ group (Fig. 4O). In addition, we further examined the spine density of hippocampal neurons and observed a significant increase in the dendritic spine density of the Pre group when compared with the SZ rats (Fig. 4P, Q). Together, these findings strongly support that $\omega$-3PUFAs prevent hippocampal neuronal damage in MK801-induced SZ.

\section{w-3PUFAs Prevented the Decreased CREB Ser133 Phosphorylation Induced by MK801}

An increasing number of studies reported that supplementation with long-chain polyunsaturated fatty acids is beneficial in promoting brain and neuronal development, and improving cognitive function [36]; however, the mechanism remains to be elucidated. We have previously found that MK801 induced cognitive impairments via altering the CREB/BDNF/TrkB pathway [22]. We therefore hypothesized that the $\omega$-3PUFAs might have prevented the cognitive impairments by inducing the phosphorylation of
Fig. 6 CREB S133A non-phosphorylation mimetic mutation inhibited the recovering effect of $\omega$-3PUFAs in schizophrenia. (A) Experimental design sketch. Sixty healthy SD rats were randomly divided into 4 groups. In the control, 15 rats were injected with AAV9/CTR virus in the hippocampal CA1. For the model (Mod) group, 15 rats were daily injected intraperitoneally with MK801 $(0.3 \mathrm{mg} / \mathrm{kg})$ for 1 week. The preventive (Pre) group are 15 rats with a 4-week intragastric gavage of fish oil $(400 \mathrm{mg} / \mathrm{kg})$ and intraperitoneal daily injection of MK801 $(0.3 \mathrm{mg} / \mathrm{kg})$ for 1 week. The remaining 15 rats (S133A-Pre group) were injected with AAV9/CREB-S133A virus in the hippocampal CA1 and were intragastrically administered fish oil $(400 \mathrm{mg} / \mathrm{kg})$ for 4 weeks and intraperitoneally daily injected with MK801 $(0.3 \mathrm{mg} / \mathrm{kg})$ for the last 1 week. Following the treatment, behavioral, electrophysiological, and biochemical tests were performed. (B) AAV9/S133A was injected into the hippocampal CA1 region of the SD rats, and GFP expression was observed (green) 4 weeks after injection. (C) Novel object recognition test (NOR) showing the time spent exploring the new object in $2 \mathrm{~h}$ and $24 \mathrm{~h}$. (D) Morris water maze test: the spatial memory was tested by removing the platform, and the time spent in the target quadrant and the number of target platform crossing were measured. (E) Hippocampal CA3-CA1 LTP and its quantification (F) were recorded by using the MED64 system. Normalized CA3-CA1 fEPSP mean slope recorded from the CA1 dendritic region in hippocampal slices. $(\mathrm{G}, \mathrm{H})$ Representative dendritic spines of neurons from Golgi impregnated hippocampus (G), averaged spine density (mean spine number per $10-\mu \mathrm{m}$ dendrite segment) was measured (H). Scale bar $=2 \mu \mathrm{m}$. (I-K) Brain tissues (hippocampus) were homogenized, and $\mathrm{p}$-TrkB, TrkB, and BDNF protein levels were detected by immunoblotting. Actin was used as a loading control. Quantitative analysis of BDNF (J), p-TrkB (K) levels. $n=4$ for all groups, ${ }^{*} p<0.05$, $* * p<0.01, * * * p<0.001$ versus AAV9/CTR or preventive groups

the CREB at Ser133 site, thereby activating the CREB/BDNF/TrkB pathway. As expected, the western blot result analysis showed a significant increase in BDNF and $p$-CREB, $p$-TrkB following $\omega$-3PUFA supplementation when compared with the model MK801-treated group (Fig. 5A-D). These data suggest that $\omega$-3PUFAs

\section{a}

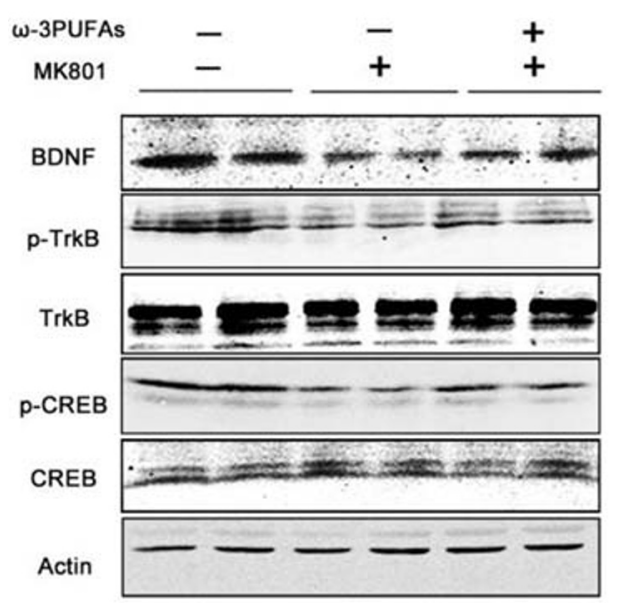

b

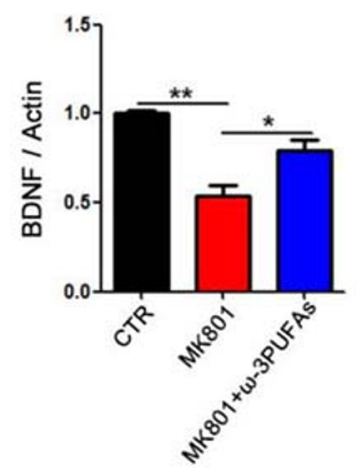

C

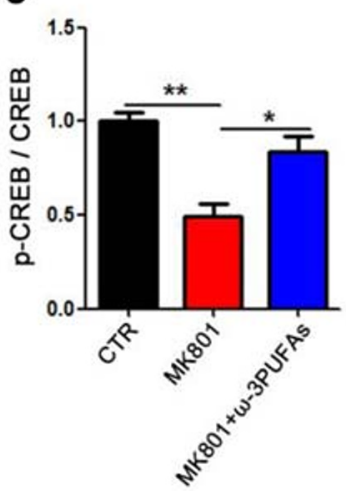

d

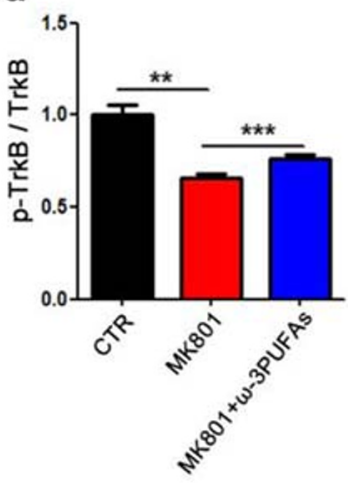

Fig. $5 \omega$-3PUFAs prevented the decrease of CREB Ser133 phosphorylation induced by MK801 and upregulated BDNF/TrkB pathway. (A-D) Brain tissues (hippocampus) were homogenized, and pTrkB, TrkB, p-CREB, CREB, and BDNF protein levels were detected by immunoblotting. Actin was used as a loading control. Quantitative analysis of the BDNF (B), p-CREB (C), and p-TrkB (D) levels. $n=4$ for all groups; data are presented as mean \pm SEM. $* p<0.05$, $* * p<0.01, * * * p<0.001$ versus model group 


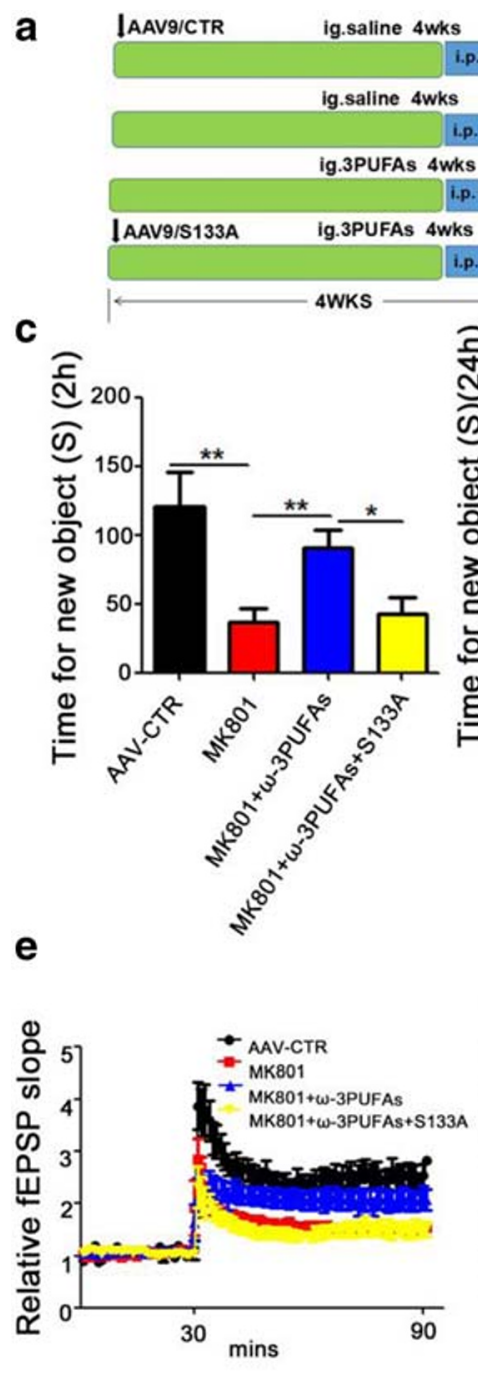

b

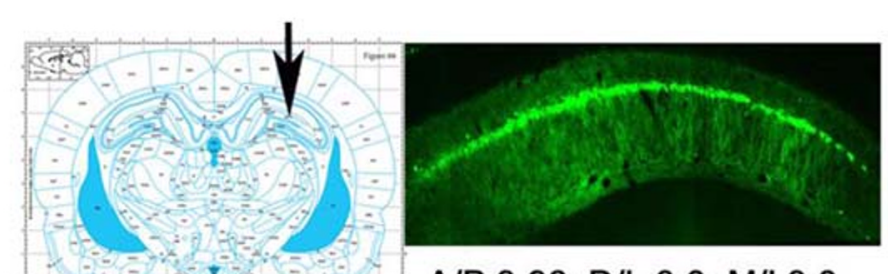

A/P 3.96; D/L 3.0; M/L3.0
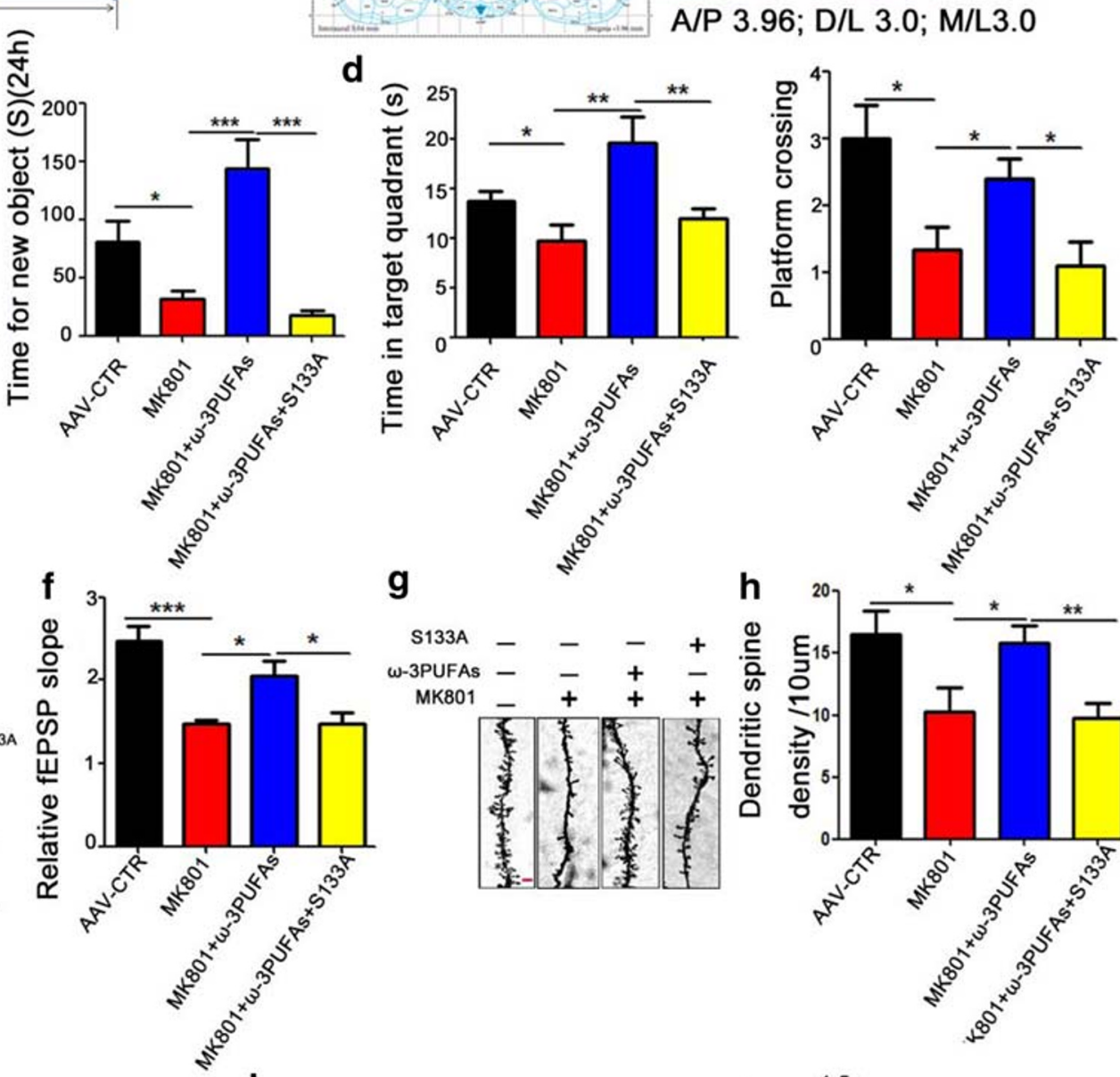

g
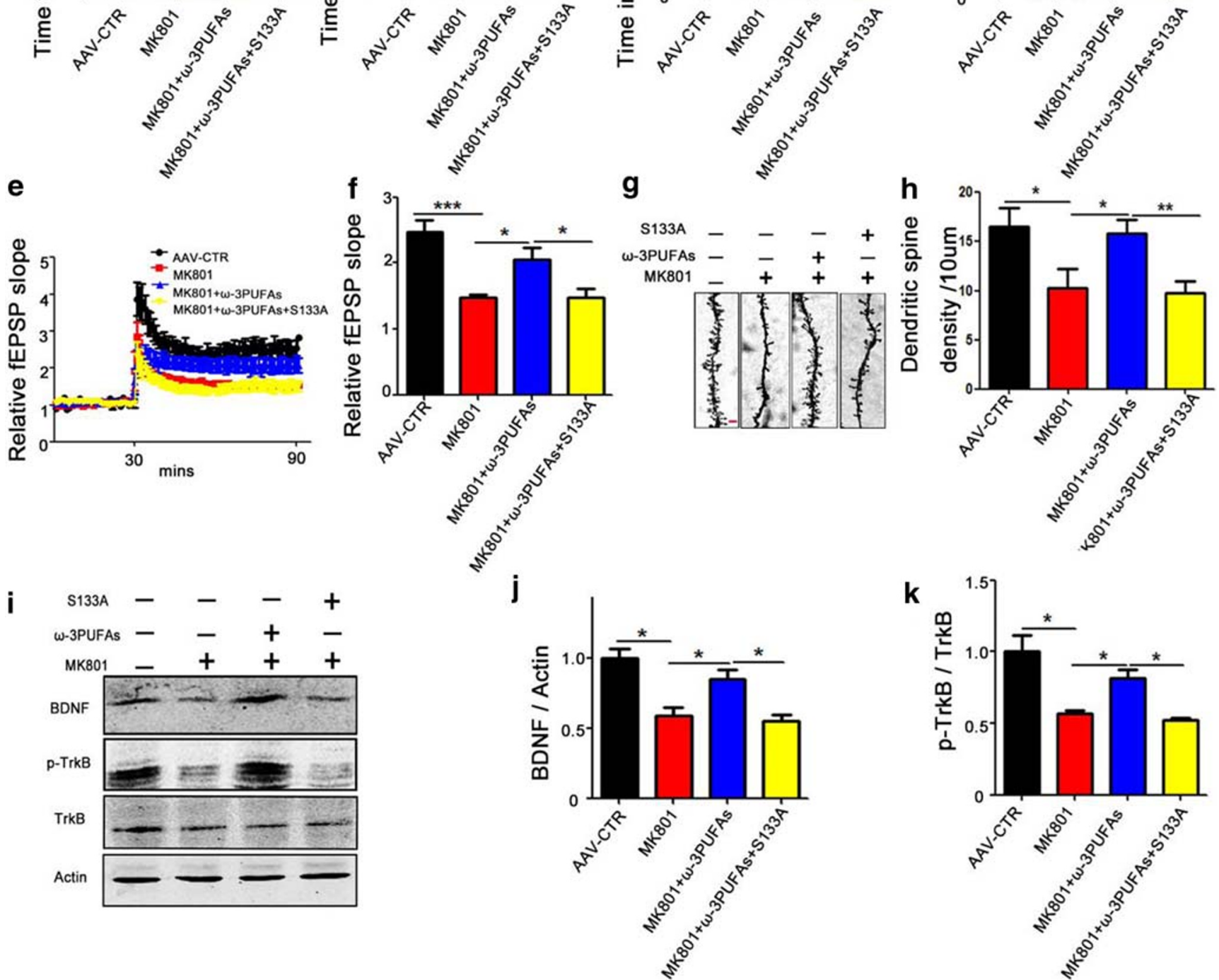

might upregulate BDNF/TrkB pathway via an increase in CREB phosphorylation at Ser133 in SZ.

To investigate the effect of $\omega$-3PUFAs on cognition and the changes of signaling molecules at the administration of $\omega$ -
3PUFAs in normal untreated animals, we firstly performed behavior test (sFig. 1A). NOR test showed a significant increase in the curiosity toward new things in the $\omega$-3PUFA group when compared with the normal untreated rats (sFig. 


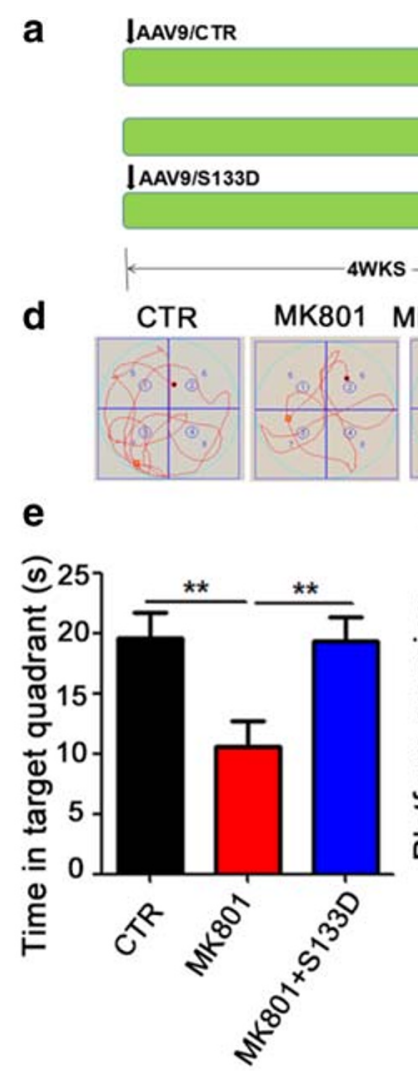

i

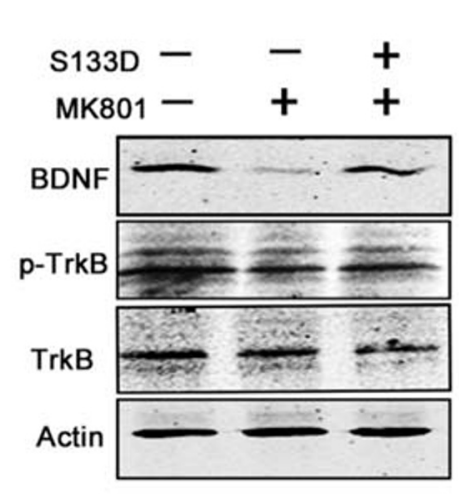

I

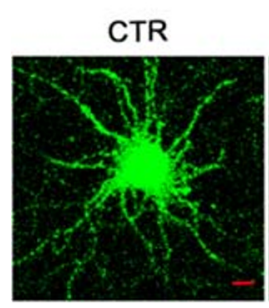

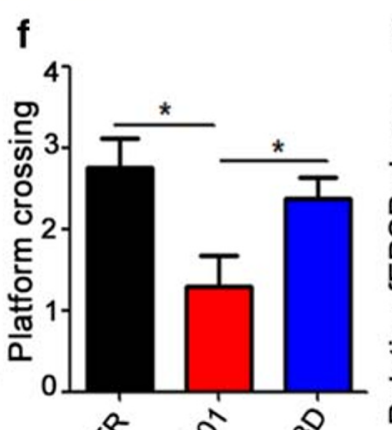

से b
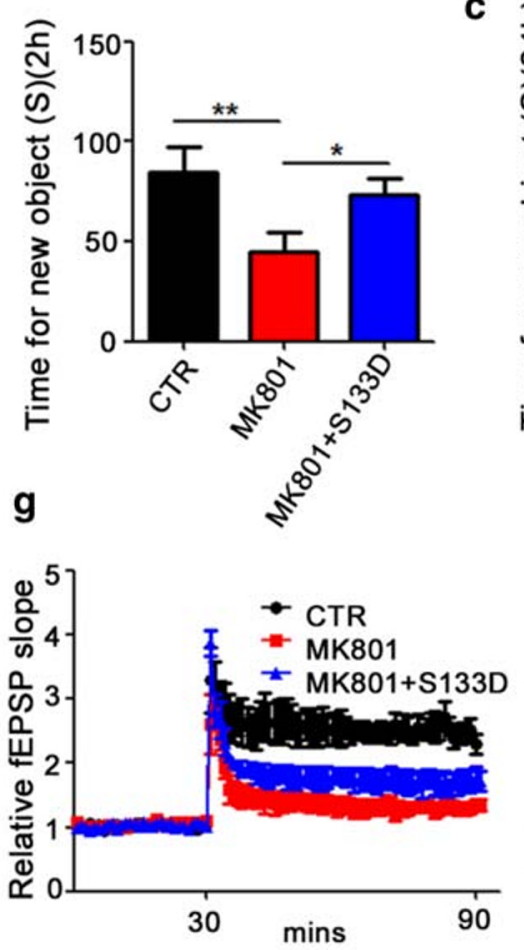

j

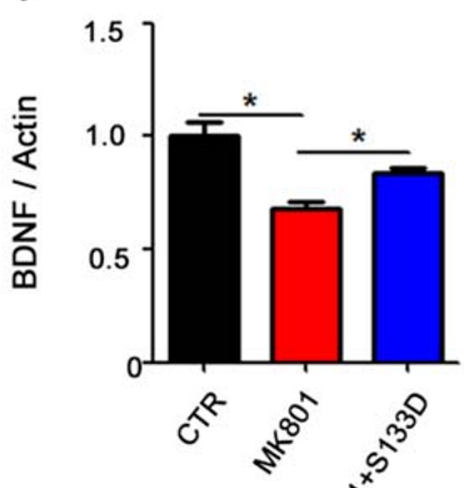

m

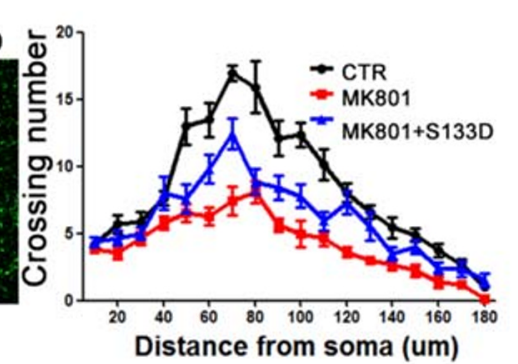

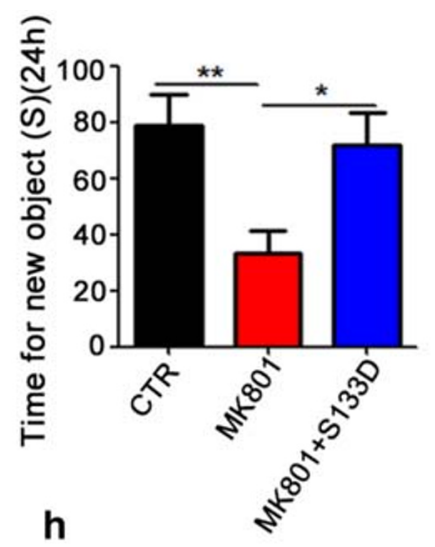

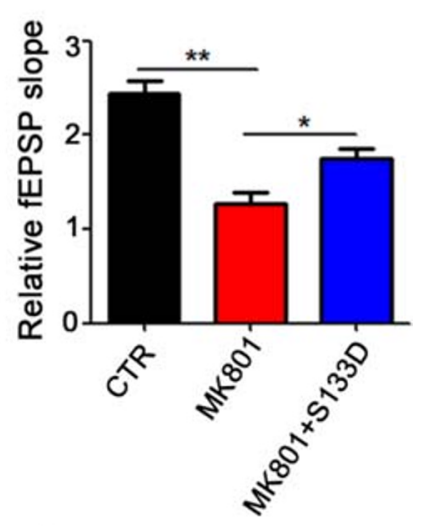

k
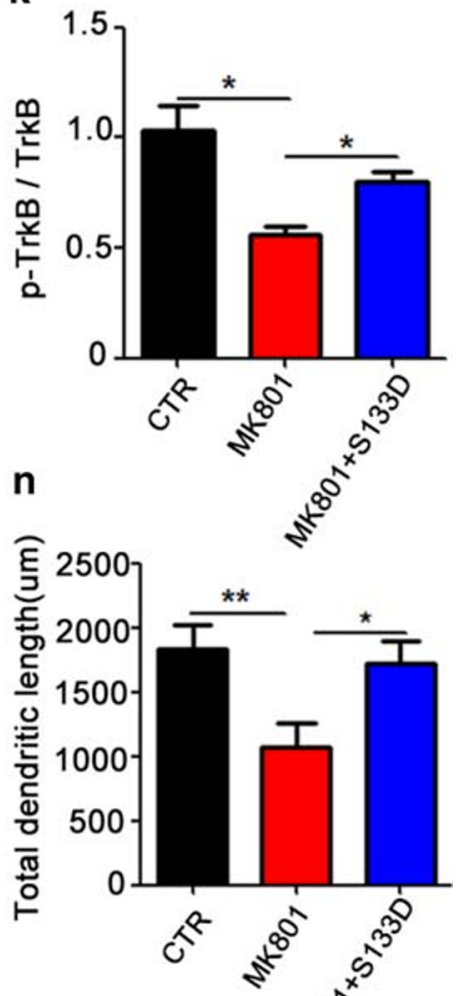
Fig. 7 CREB S133D phosphorylation mimetic mutation attenuated the cognitive and synaptic plasticity alterations in MK801-induced schizophrenia. (A) Experimental design sketch. Forty-five healthy SD rats were randomly divided into 3 groups. In the first group (control), 15 rats were injected with AAV9/CTR virus in hippocampal CA1 and allowed for 4 weeks expression. Another 15 rats were daily injected intraperitoneally with MK801 $(0.3 \mathrm{mg} / \mathrm{kg})$ for 1 week to serve as model (Mod) group, while the last 15 rats were injected of AAV9/CREB-S133D virus in hippocampal CA1 and at the same time were intraperitoneally daily injected with MK801 $(0.3 \mathrm{mg} / \mathrm{kg})$ for 1 week to serve as the S133Dtherapy group. Following the treatment, behavioral, electrophysiological, and biochemical tests were performed. (B, C) Novel object recognition test (NOR) shows the time spent exploring new object in $2 \mathrm{~h}(\mathrm{~B})$ and $24 \mathrm{~h}$ (C). (D-F) Morris water maze test: the spatial memory was tested by removing the platform, the representative traces searching the removed platform on day 6 of the training (D), the time spent in the target quadrant (E) and number of the position of the platform crossings (F) were shown. (G, H) Hippocampal CA3-CA1 LTP was recorded by using the MED64 system. Normalized CA3-CA1 fEPSP mean slope recorded from the CA1 dendritic region in hippocampal slices. (I-K) brain tissues (hippocampus) were homogenized, and BDNF, p-TrkB, and TRK protein levels were detected by immunoblotting. Actin was used as a loading control. Quantitative analysis of the BDNF (J), and p-TrkB (K) levels. (L-N) Rat primary hippocampal neurons at DIV 9 were treated with DMSO, MK801, and AAV9/CREB-S133D+MK801, and representative images after treatment (L), Sholl analysis (M), quantitative analysis of dendritic length $(N)$, scale bar $=10 \mu \mathrm{m} . n=16$ hippocampal neurons. All data represent as mean \pm SEM. ${ }^{*} p<0.05, * * p<0.01$, and $* * * p<0.001$ versus model

1B-C). Moreover, the electrophysiology experiments showed that $\omega$-3PUFAs enhanced the slope of field excitatory postsynaptic potential (fEPSP) after high-frequency stimulation (HFS) compared with the normal control group (sFig. 1DE). Next, we also performed Western blotting (sFig. 1F-I) and the result showed a significant increase in BDNF and pCREB in $\omega$-3PUFA group compared with the control group, while there was an upward trend in p-TrkB with no statistical difference. Together, these data suggest that $\omega$-3PUFAs might be beneficial to cognitive function by upregulating BDNF/ CREB pathway and synaptic plasticity.

\section{CREB S133A Abolished the Preventive Effects of $\omega$-3PUFAs in MK801-Induced SZ}

We have shown that $\omega$-3PUFAs could improve MK801induced cognitive dysfunction, an effect that may be mediated via preventing or recovering the MK801-induced decreased Ser133 phosphorylation/activation of CREB. To further confirm this hypothesis, we performed a non-phosphorylation mimic CREB (S133A, an inactivated mutation) at the Ser133 site to investigate the mechanism. Sixty healthy SD rats were randomly divided into 4 groups and treated as shown in (Fig. 6A). In the control, 15 rats were injected with AAV9/ CTR virus in the hippocampal CA1 (Fig. 6B). For the model (Mod) group, 15 rats were daily injected intraperitoneally with MK801 $(0.3 \mathrm{mg} / \mathrm{kg})$ for 1 week. The preventive (Pre) group are 15 rats with a 4-week intragastric gavage of fish oil $(400 \mathrm{mg} / \mathrm{kg})$ and intraperitoneal daily injection of MK801 $(0.3 \mathrm{mg} / \mathrm{kg})$ for 1 week. The remaining 15 rats (S133A-Pre group) were injected with AAV9/CREB-S133A virus in the hippocampal CA1 and were intragastrically administered fish oil $(400 \mathrm{mg} / \mathrm{kg})$ for 4 weeks and intraperitoneally daily injected with MK801 $(0.3 \mathrm{mg} / \mathrm{kg})$ for the last 1 week. Following the treatment, we performed a set of behavioral tests. NOR test showed a significant decrease in the curiosity toward new things in the S133A-Pre group when compared with the Pre group, as suggested by the decrease in the time spent for the exploration of new object within $2 \mathrm{~h}$ and $24 \mathrm{~h}$ (Fig. 6C). Moreover, MWM showed a remarkable decrease in both the time spent in the target quadrant and the number of target platform crossing in the S133A-Pre group as compared to the Pre group (Fig. 6D). In view of the above data, we wonder whether the Ser133 residue of CREB plays a role in Pre group synaptic plasticity. We therefore carried out electrophysiology experiments and found that $\omega$-3PUFAs enhanced the slope of field excitatory postsynaptic potential (fEPSP) after high-frequency stimulation (HFS) compared with the SZ Mod group (Fig. 6E). Interestingly, we observed that in the S133A-Pre group, the slope of field excitatory postsynaptic potential (fEPSP) was lower than that in the Pre group (Fig. 6F). In addition, we further examined the spine density of hippocampal neurons (Fig. 6G). The Golgi staining showed a significant decrease in the dendritic spine density of the S133A-Pre rats compared with the Pre group (Fig. 6H). We also performed Western blotting (Fig. 6I-K) and the result showed a significant decrease in BDNF (Fig. 6J) and p-TrkB (Fig. 6K) in the S133A-Pre group compared with the Pre group, further suggesting the importance of CREB Ser133 in synaptic plasticity. These results together imply that Ser133 phosphorylation of the CREB is required for the $\omega$-3PUFAmediated beneficial effect in MK801-induced SZ.

\section{CREB S133D Improved Cognitive Dysfunction in MK801-Induced Schizophrenia}

To test whether CREB Ser133 is a therapeutic site for SZinduced cognitive impairments, we also performed a phosphorylation mimic (S133D, an activated mutation) at the CREB Ser133 site. We used 45 healthy SD rats randomly divided into 3 groups. In the first group (control), 15 rats were injected with AAV9/CTR virus in hippocampal CA1 and allowed for 4 weeks expression. Another 15 rats were daily injected intraperitoneally with MK801 $(0.3 \mathrm{mg} / \mathrm{kg})$ for 1 week to serve as model (Mod) group, while the last 15 rats were injected of AAV9/CREB-S133D virus in hippocampal CA1 and at the same time were intraperitoneally daily injected with MK801 $(0.3 \mathrm{mg} / \mathrm{kg})$ for 1 week to serve as the S133D-therapy group, and allowed for a total period of 4 weeks (Fig. 7A). Following the treatment, we again performed a couple of 
behavioral tests. The results of the NOR showed that in the S133D group, the curiosity for exploring new things was significantly increased, as indicated by the increased time spent discovering a new object within $2 \mathrm{~h}$ (Fig. 7B) and $24 \mathrm{~h}$ (Fig. 7C) when compared with the Mod group. Similarly, the MWM (Fig. 7D) revealed a remarkably increased time in the target quadrant (Fig. 7E) and increased number of target platform crossing (Fig. 7F) in the S133D group. Moreover, the fEPSP slope following HFS was found to be higher in S133D rats compared with the MK801 group (Fig. 7G, H). Western blotting (Fig. 7I) further validated this result as it shows a significant increase in BDNF (Fig. 7J) and p-TrkB (Fig. 7K) in the S133D group compared with the Mod group. Similarly, hippocampal primary neurons were transduced with AAV9/ CREB-S133D virus and treated with MK801 on the ninth day, and AAV9/GFP was used to examine dendritic morphology (Fig. 7L). CREB-S133D resulted in an obvious increase in the dendritic complexity at all points farther than $60 \mu \mathrm{m}$ from the cell body when compared with MK801 treated neurons (Fig. $7 \mathrm{M}, \mathrm{N})$. These data indicated that the dephosphorylation of Ser133 residue of CREB is the mechanism whereby MK801 mediates its deleterious effect in the MK801-induced SZ, supporting CREB Ser133 might be a potential therapeutic site for SZ-induced cognitive alterations.

\section{Discussion}

Schizophrenia is a complex condition associated with cognitive impairments $[37,38]$, and is increasingly becoming more prevalent with its burden more and more heavy in China [39, 40] and the world [41]. Its etiopathogenesis is still unclear and the underlying molecular mechanisms remain unknown, making its treatment and preventive measures very limited, imposing a heavy burden on patients and their families. Therefore, actively exploring the molecular mechanism of cognitive impairments in schizophrenia will pave the way toward the preventive and treatment measures which are the fundamental ways to improve the quality of life of patients.

Cognitive impairments are more likely to affect the prognosis outcome than psychotic symptoms, which impair the professional social activities of patients and seriously affecting their quality of life [42]. Thus, improving the cognitive function becomes imperative as it may contribute to the treatment and prevention of schizophrenia. $\omega$-3PUFA is an essential nutrient and has been shown to improve cognitive impairments in Alzheimer disease (AD) patients [43]. So, we wondered whether $\omega$-3PUFAs can improve the cognitive function in SZ and play a role in prevention. In this study, we investigated the preventive effect of $\omega$-3PUFAs in MK801-induced $\mathrm{SZ}$ in rats and its related mechanisms. We found that MK801 leads to the impairments of learning and memory ability of the injected rats and that $\omega$-3PUFAs can improve cognitive dysfunction by antagonizing the MK801-induced SZ-like alterations. BDNF is an important neurotrophin that play a crucial role in the brain development and involved in learning and memory via the CREB/BDNF/TrkB pathway [22], in which BDNF binding to TrkB enhances itself autophosphorylation $[33,34]$ and in turn activates the Ras-MAPK pathway, and finally activates CREB by inducing its phosphorylation at the Ser133 site. CREB activation promotes synaptic plasticity and neurogenesis by increasing the expression of the BDNF gene [35]. Moreover, $\omega$-3PUFAs can upregulate CREB phosphorylation which regulates the expression of multiple proteins involved in memory [23]. In the present study, we found MK801-induced SZ could cause cognitive impairments accompanying with CREB/BDNF/TrkB pathway dysfunction, BDNF deficiency, and synaptic dysfunction, all of which were abrogated by $\omega$-3PUFAs, suggesting that downregulation of CREB/BDNF/TrkB pathway might mediate SZ-associated cognitive impairments. We therefore further focus on the CREB/BDNF/TrkB pathway. Our study demonstrated that MK801 reduces the protein level of $\mathrm{p}$-CREB resulting in a subsequent downregulation of BDNF and p-TrkB in the hippocampus of injected rats. Interestingly, these effects were prevented by prior administration of $\omega$-3PUFAs. CREB phosphorylation at Ser133 is crucial for its activation and the downstream effects. We thus used the AAV9/CREB-S133A (non-phosphorylation mutation) to inject the rats in the hippocampal CA1 region and found out that the nonphosphorylated CREB abolished the recovery beneficial effect of $\omega$-3PUFAs, resulting in behavioral and cognitive impairments, and decrease protein levels of BDNF and p-TrkB. Moreover, Golgi staining revealed a decrease in the total density of dendritic spines following AAV9/CREB-S133A injection in the rats. Next, we also used AAV9/CREB-S133D (phosphorylation mutation) to inject the hippocampal CA1 region, and discovered that this effectively improved the cognitive dysfunction and increased the protein level of both BDNF and p-TrkB. In addition, AAV9/GFP immunofluorescence staining showed an increase in the dendritic complexity in the AAV9/CREB-S133D-transduced primary hippocampal neurons. In conclusion, our study demonstrates that $\omega$ 3PUFAs regulate $\mathrm{CREB} / \mathrm{BDNF} / \mathrm{TrkB}$ pathway by inducing the phosphorylation of CREB Ser133, thereby activating CREB which leads to increased synaptic plasticity, and reduced hippocampal neuronal loss, and thus antagonizes the MK801-induced SZ cognition dysfunction in rats. This study provides a basis of the molecular mechanism associated with memory and cognitive impairments in MK801-induced SZ and gives an insight for the treatment and prevention of SZ by $\omega$-3PUFAs.

Acknowledgments This work was supported in parts by grants from the National Natural Science Foundation of China (31771114, 81771445, 31929002, and 81571255); grant from the Innovative Research Groups 
of the National Natural Science Foundation of China (81721005); grant from the Ministry of Science and Technology of China (2016YFC1305800); project funded by the China Postdoctoral Science Foundation, grant no. 2019 T120662; and the Academic Frontier Youth Team Project to Xiaochuan Wang from Huazhong University of Science and Technology. All the authors wish to express their gratitude and appreciation to Dan Ke and Qun Wang for their expertise and technical assistance. Thanks to all the members of our research team for the constructive discussion and suggestions that shaped this work.

Author Contributions XCW planned, organized, and designed all experiments and results, including the writing of the manuscript. CG and YL planned and performed all experiments. YL, WL, YARM, YX, KZ, YX, and DK assisted with the manuscript preparation. RL, JZW, and HS analyzed the data. XS and MF analyzed and interpreted the data. All authors read and approved the final manuscript.

Availability of Data and Materials The datasets used and/or analyzed during the present study are available from the corresponding author on reasonable request.

\section{Compliance with Ethical Standards}

Conflict of Interest The authors declare that they have no conflict of interest.

Ethics Approval and Consent to Participate No humans were used in this research. All animal experiments were approved by the Animal Care and Use Committee of Huazhong University of Science and Technology, and performed in compliance with the National Institutes of Health Guide for the Care and Use of Laboratory Animals.

Consent for Publication All authors consented to publication of this paper.

Abbreviations SZ, schizophrenia; $\omega$-3PUFAs, $\omega$-3 polyunsaturated fatty acids; BDNF, brain-derived neurotrophic factor; CREB, cAMP response element binding protein; NMDAR, N-methyl-D-aspartic acid receptor; AMAPR, $\alpha$-amino-3-hydroxy-5-methyl-4-isoxazole-propionic acid receptor; $\mathrm{AAV}$, adeno-associated virus; Ctr, control; Mod, model; Pre, preventive; MWM, Morris water maze; NOR, novel object recognition; SC, Schaffer collateral; PPR, paired pulse ratio; sEPSCs, spontaneous excitatory postsynaptic currents; fEPSP, field excitatory postsynaptic potential; LTP, long-term potentiation

\section{References}

1. Buck B, Gagen EC, Luther L, Kukla M, Lysaker PH. Dynamic relationships between emotional distress, persecutory ideation, and metacognition in schizophrenia. J Clin Psychol 2019.

2. Wedervang-Resell K, Friis S, Lonning V, Smelror RE, Johannessen $\mathrm{C}$, Reponen EJ, et al. Increased interleukin 18 activity in adolescents with early-onset psychosis is associated with cortisol and depressive symptoms. Psychoneuroendocrinology 2019:104513.

3. MacDougall AG, Price E, Vandermeer MRJ, Lloyd C, Bird R, Sethi $\mathrm{R}$, et al. Youth-focused group mindfulness-based intervention in individuals with early psychosis: A randomized pilot feasibility study. Early Interv Psychiatry 2019;13(4):993-8.

4. Mariano MA, Tang K, Kurtz M, Kates WR. Examining the durability of a hybrid, remote and computer-based cognitive remediation intervention for adolescents with 22q11.2 deletion syndrome. Early Interv Psychiatry 2018;12(4):686-93.
5. Guo JY, Ragland JD, Carter CS. Memory and cognition in schizophrenia. Mol Psychiatry 2019;24(5):633-42.

6. Enwright Iii JF, Huo Z, Arion D, Corradi JP, Tseng G, Lewis DA. Transcriptome alterations of prefrontal cortical parvalbumin neurons in schizophrenia. Mol Psychiatry 2018;23(7):1606-13.

7. Takahashi M, Shirakawa O, Toyooka K, Kitamura N, Hashimoto T, Maeda K, et al. Abnormal expression of brain-derived neurotrophic factor and its receptor in the corticolimbic system of schizophrenic patients. Mol Psychiatry 2000;5(3):293-300.

8. Rizos EN, Papathanasiou M, Michalopoulou PG, Mazioti A, Douzenis A, Kastania A, et al. Association of serum BDNF levels with hippocampal volumes in first psychotic episode drug-naive schizophrenic patients. Schizophr Res 2011;129(2-3):201-4.

9. Lu B, Nagappan G, Guan X, Nathan PJ, Wren P. BDNF-based synaptic repair as a disease-modifying strategy for neurodegenerative diseases. Nat Rev Neurosci 2013;14(6):401-16.

10. Nagahara AH, Tuszynski MH. Potential therapeutic uses of BDNF in neurological and psychiatric disorders. Nat Rev Drug Discov 2011;10(3):209-19.

11. Minichiello L. TrkB signalling pathways in LTP and learning. Nat Rev Neurosci 2009;10(12):850-60.

12. Angelucci F, Brene S, Mathe AA. BDNF in schizophrenia, depression and corresponding animal models. Mol Psychiatry 2005;10(4): 345-52.

13. Green MJ, Matheson SL, Shepherd A, Weickert CS, Carr VJ. Brain-derived neurotrophic factor levels in schizophrenia: a systematic review with meta-analysis. Mol Psychiatry 2011;16(9): 960-72.

14. Gomez-Pinilla F, Tyagi E. Diet and cognition: interplay between cell metabolism and neuronal plasticity. Curr Opin Clin Nutr Metab Care 2013;16(6):726-33.

15. Freeman MP, Rapaport MH. Omega-3 fatty acids and depression: from cellular mechanisms to clinical care. J Clin Psychiatry 2011;72(2):258-9.

16. Hirashima F, Parow AM, Stoll AL, Demopulos CM, Damico KE, Rohan ML, et al. Omega-3 fatty acid treatment and T(2) whole brain relaxation times in bipolar disorder. Am J Psychiatry 2004;161(10):1922-4.

17. DeCarolis NA, Eisch AJ. Hippocampal neurogenesis as a target for the treatment of mental illness: a critical evaluation. Neuropharmacology 2010;58(6):884-93.

18. Wolf RC, Hose A, Frasch K, Walter H, Vasic N. Volumetric abnormalities associated with cognitive deficits in patients with schizophrenia. Eur Psychiatry 2008;23(8):541-8.

19. Agrawal R, Gomez-Pinilla F. 'Metabolic syndrome' in the brain: deficiency in omega-3 fatty acid exacerbates dysfunctions in insulin receptor signalling and cognition. J Physiol 2012;590(10):2485-99.

20. Andreeva VA, Kesse-Guyot E, Barberger-Gateau P, Fezeu L, Hercberg S, Galan P. Cognitive function after supplementation with $\mathrm{B}$ vitamins and long-chain omega-3 fatty acids: ancillary findings from the SU.FOL.OM3 randomized trial. Am J Clin Nutr 2011;94(1):278-86.

21. English JA, Harauma A, Focking M, Wynne K, Scaife C, Cagney G, et al. Omega-3 fatty acid deficiency disrupts endocytosis, neuritogenesis, and mitochondrial protein pathways in the mouse hippocampus. Front Genet 2013;4:208.

22. Fang MS, Li X, Qian H, Zeng K, Ye M, Zhou YJ, et al. omega3PUFAs prevent MK-801-induced cognitive impairment in schizophrenic rats via the CREB/BDNF/TrkB pathway. J Huazhong Univ Sci Technolog Med Sci 2017;37(4):491-5.

23. Gao H, Yan P, Zhang S, Huang H, Huang F, Sun T, et al. Long-Term Dietary Alpha-Linolenic Acid Supplement Alleviates Cognitive Impairment Correlate with Activating Hippocampal CREB Signaling in Natural Aging Rats. Mol Neurobiol. 2016;53(7): 4772-86. 
24. Williams CM, El Mohsen MA, Vauzour D, Rendeiro C, Butler LT, Ellis JA, et al. Blueberry-induced changes in spatial working memory correlate with changes in hippocampal CREB phosphorylation and brain-derived neurotrophic factor (BDNF) levels. Free Radic Biol Med 2008;45(3):295-305.

25. Shin E, Kashiwagi Y, Kuriu T, Iwasaki H, Tanaka T, Koizumi H, et al. Doublecortin-like kinase enhances dendritic remodelling and negatively regulates synapse maturation. Nat Commun 2013;4: 1440.

26. Han RZ, Hu JJ, Weng YC, Li DF, Huang Y. NMDA receptor antagonist MK-801 reduces neuronal damage and preserves learning and memory in a rat model of traumatic brain injury. Neurosci Bull 2009;25(6):367-75.

27. Li C, Tang Y, Yang J, Zhang X, Liu Y, Tang A. Sub-chronic Antipsychotic Drug Administration Reverses the Expression of Neuregulin 1 and ErbB4 in a Cultured MK801-Induced Mouse Primary Hippocampal Neuron or a Neurodevelopmental Schizophrenia Model. Neurochem Res 2016;41(8):2049-64.

28. Xiu Y, Kong XR, Zhang L, Qiu X, Chao FL, Peng C, et al. White matter injuries induced by MK-801 in a mouse model of schizophrenia based on NMDA antagonism. Anat Rec (Hoboken) 2014;297(8):1498-507.

29. Myint L, Wang R, Boukas L, Hansen KD, Goff LA, Avramopoulos D. A screen of 1,049 schizophrenia and 30 Alzheimer's-associated variants for regulatory potential. Am J Med Genet B Neuropsychiatr Genet 2019

30. DeMichele-Sweet MAA, Weamer EA, Klei L, Vrana DT, Hollingshead DJ, Seltman HJ, et al. Correction: Genetic risk for schizophrenia and psychosis in Alzheimer disease. Mol Psychiatry 2019.

31. Trost W, Fruhholz S. The hippocampus is an integral part of the temporal limbic system during emotional processing: Comment on "The quartet theory of human emotions: An integrative and neurofunctional model" by S. Koelsch et al. Phys Life Rev 2015;13:87-8

32. Bartsch T, Wulff P. The hippocampus in aging and disease: From plasticity to vulnerability. Neuroscience 2015;309:1-16.

33. Choo M, Miyazaki T, Yamazaki M, Kawamura M, Nakazawa T, Zhang J, et al. Retrograde BDNF to TrkB signaling promotes synapse elimination in the developing cerebellum. Nat Commun 2017;8(1):195.

34. Harward SC, Hedrick NG, Hall CE, Parra-Bueno P, Milner TA, Pan $\mathrm{E}$, et al. Autocrine BDNF-TrkB signalling within a single dendritic spine. Nature 2016;538(7623):99-103.
35. Mehrafza S, Kermanshahi S, Mostafidi S, Motaghinejad M, Motevalian M, Fatima S. Pharmacological evidence for lithiuminduced neuroprotection against methamphetamine-induced neurodegeneration via Akt-1/GSK3 and CREB-BDNF signaling pathways. Iran J Basic Med Sci 2019;22(8):856-65.

36. He C, Qu X, Cui L, Wang J, Kang JX. Improved spatial learning performance of fat-1 mice is associated with enhanced neurogenesis and neuritogenesis by docosahexaenoic acid. Proc Natl Acad Sci U S A 2009;106(27):11370-5.

37. Ma X, Zheng W, Li C, Li Z, Tang J, Yuan L, et al. Decreased regional homogeneity and increased functional connectivity of default network correlated with neurocognitive deficits in subjects with genetic high-risk for schizophrenia: A resting-state fMRI study. Psychiatry Res 2019;281:112603.

38. Friedman JI, Vrijenhoek T, Markx S, Janssen IM, van der Vliet WA, Faas BH, et al. CNTNAP2 gene dosage variation is associated with schizophrenia and epilepsy. Mol Psychiatry 2008;13(3):261-6.

39. Xu DR, Xiao S, He H, Caine ED, Gloyd S, Simoni J, et al. Lay health supporters aided by mobile text messaging to improve adherence, symptoms, and functioning among people with schizophrenia in a resource-poor community in rural China (LEAN): A randomized controlled trial. PLoS Med 2019;16(4):e1002785.

40. Wong AWS, Ting KT, Chen EYH. Group cognitive behavioural therapy for Chinese patients with psychotic disorder: A feasibility controlled study. Asian J Psychiatr 2019;39:157-64.

41. Bruno A, Pandolfo G, Crucitti M, Troili GM, Battaglia F, Zoccali RA, et al. Effect of Red Yeast Rice on Cognitive Functioning in Schizophrenia: Data From a Pilot Study. J Clin Psychopharmacol 2019;39(3):210-3.

42. Bell M, Bryson G, Greig T, Corcoran C, Wexler BE. Neurocognitive enhancement therapy with work therapy: effects on neuropsychological test performance. Arch Gen Psychiatry 2001;58(8):763-8.

43. Belkouch M, Hachem M, Elgot A, Lo Van A, Picq M, Guichardant $\mathrm{M}$, et al. The pleiotropic effects of omega-3 docosahexaenoic acid on the hallmarks of Alzheimer's disease. J Nutr Biochem 2016;38: $1-11$.

Publisher's Note Springer Nature remains neutral with regard to jurisdictional claims in published maps and institutional affiliations. 